\title{
(W)WILEY
}

\section{Increased expression of colony-stimulating factor-1 in mouse spinal cord with experimental autoimmune encephalomyelitis correlates with microglial activation and neuronal loss}

\begin{tabular}{|c|c|}
\hline Journal: & GLIA \\
\hline Manuscript ID & GLIA-00262-2017.R1 \\
\hline Wiley - Manuscript type: & Original Research Article \\
\hline Date Submitted by the Author: & $\mathrm{n} / \mathrm{a}$ \\
\hline Complete List of Authors: & $\begin{array}{l}\text { Gushchina, Svetlana; Queen Mary, University of London, Centre for } \\
\text { Neuroscience and Trauma; Mordovskij gosudarstvennyj universitet, } \\
\text { Department of Cytology, Histology and Embryology } \\
\text { Pryce, Gareth; Queen Mary, University of London, Centre for Neuroscience } \\
\text { and Trauma } \\
\text { Yip, Ping; Queen Mary, University of London, Centre for Neuroscience and } \\
\text { Trauma } \\
\text { Wu, Dongsheng; Queen Mary University of London, School of Engineering } \\
\text { and Materials Science } \\
\text { Pallier, Patrick; Queen Mary University of London, Centre for Neuroscience } \\
\text { and Trauma } \\
\text { Giovannoni, Gavin; Queen Mary, University of London, Centre for } \\
\text { Neuroscience and Trauma } \\
\text { Baker, David; Queen Mary, University of London, Centre for Neuroscience } \\
\text { and Trauma } \\
\text { Bo, Xuenong; Queen Mary, University of London, Centre for Neuroscience } \\
\text { and Trauma }\end{array}$ \\
\hline Key Words: & $\begin{array}{l}\text { colony-stimulating factor-1, microglia, experimental autoimmune } \\
\text { encephalomyelitis, neuronal death, interleukin-34 }\end{array}$ \\
\hline
\end{tabular}




\section{Increased expression of colony-stimulating factor-1 in mouse spinal cord with} experimental autoimmune encephalomyelitis correlates with microglial activation and neuronal loss

Running title: CSF1 is upregulated in CNS of EAE mice

Svetlana Gushchina ${ }^{1,2}$, Gareth Pryce ${ }^{1}$, Ping K. Yip ${ }^{1}$, Dongsheng $\mathrm{Wu}^{3}$, Patrick Pallier ${ }^{1}$, Gavin Giovannoni ${ }^{1}$, David Baker ${ }^{1}$, and Xuenong Bo ${ }^{1}$

${ }^{1}$ Centre for Neuroscience and Trauma, Blizard Institute, Barts and the London School of Medicine and Dentistry, Queen Mary University of London, London E1 2AT, UK.

${ }^{2}$ Department of Cytology, Histology and Embryology, Ogarev Mordovia State University, Saransk 430005, Republic of Mordovia, Russia.

${ }^{3}$ School of Engineering and Materials Science, Queen Mary University of London, London E1 4NS, UK.

Correspondence: Xuenong Bo, Centre for Neuroscience and Trauma, Blizard Institute, Barts and the London School of Medicine and Dentistry, 4 Newark Street, London E1 2AT, UK. Email: x.bo@qmul.ac.uk; Telephone: +44 2078822294

Acknowledgements: The work was supported by a research grant from Foresight Inc.

Word counts: Abstract: 231; Introduction: 568; Materials and Methods: 2160; Results: 2504; Discussion: 2201; Bibliography: 1601; Figure legends: 1334; Table: 89; Total: 10862 


\begin{abstract}
Microglia contribute to pathophysiology at all stages of multiple sclerosis. Colonystimulating factor-1 (CSF1) is crucial for microglial proliferation and activation. In this study we measured the CSF1 levels and studied its cellular expression in the mouse spinal cords with experimental autoimmune encephalomyelitis (EAE) to explore the potential contribution of CSF1 in neuronal death. ELISA data showed that CSF1 levels were significantly higher in the spinal cords with acute and chronic EAE than those of normal and adjuvant-injected mice. Immunohistochemical studies demonstrated that CSF1 was expressed in astrocytes and neurons in normal mouse spinal cord. In acute EAE, CSF1 expression was significantly increased, especially in astrocytes in peripheral white matter and large motoneurons. High density of activated microglia was observed in the gray matter where motoneurons expressed high-level CSF1 in acute EAE. Significant large motoneuron loss was seen in chronic EAE and the remaining motoneurons with high-level CSF1 were enwrapped by microglia. Viral vector mediated over-expression of CSF1 in spinal neurons induced profound proliferation and activation of microglia at the injection site and microglia enwrapped CSF1-transduced neurons and their neurites. Significant loss of large CSF1-transduced neurons was seen at 2 and 3 weeks post viral injection. Demyelination in the CSF1 transduced areas was also significant. These results implicate that CSF1 upregulation in CNS may play an important role in the proliferation and activation of microglia in EAE, contributing to neuroinflammation and neurodegeneration.
\end{abstract}

Key words: colony-stimulating factor-1, interleukin-34, experimental autoimmune encephalomyelitis, microglia, neuronal death, multiple sclerosis, lentiviral vector. 
Table of Contents Image

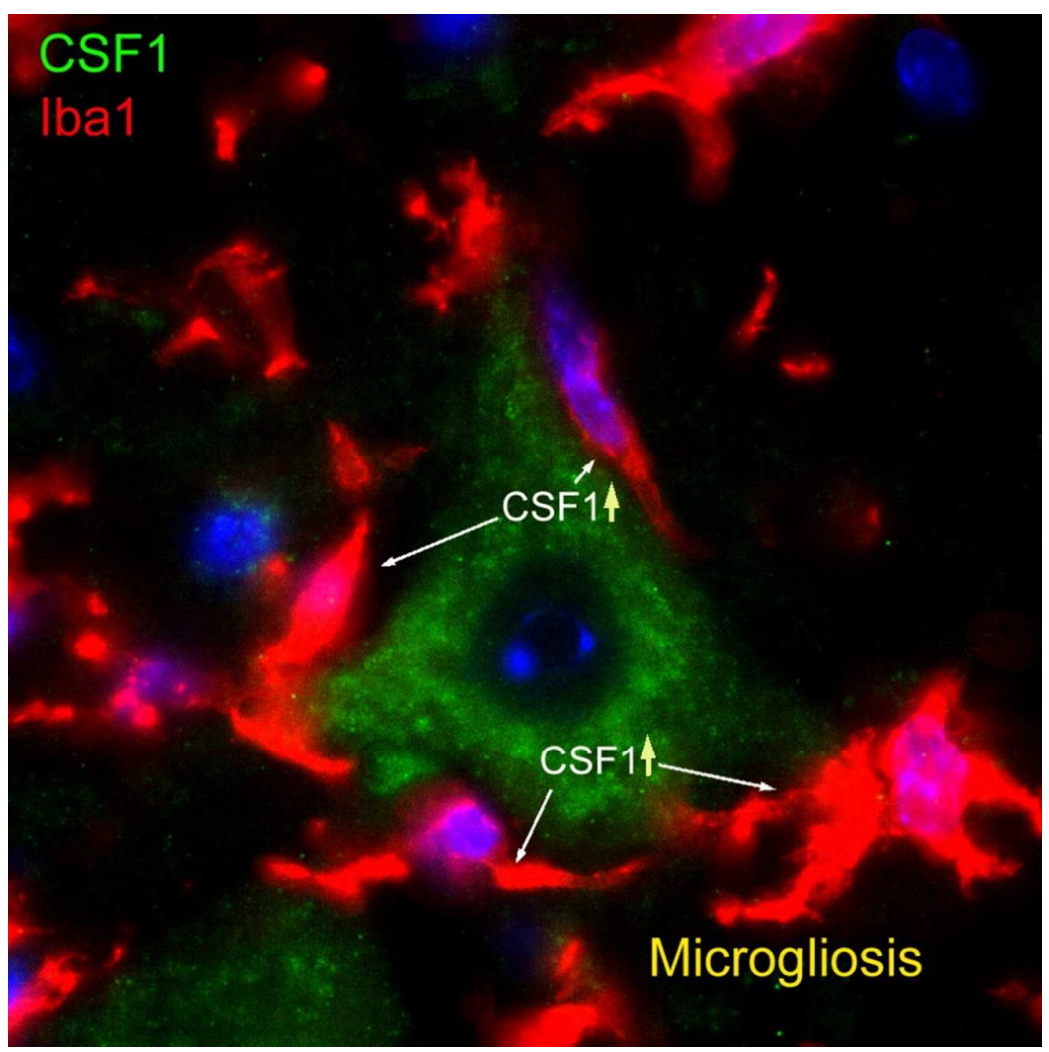




\section{Main points:}

CSF1 is significantly up-regulated in the spinal cords of acute and chronic EAE mice.

CSF1 is mainly expressed in astrocytes and neurons.

Neurons expressing high-level CSF1 are enwrapped by microglia, which may lead to neuronal death. 


\section{INTRODUCTION}

Multiple sclerosis (MS) is a neurological disorder characterized by progressive demyelination, resulting in progressive loss of motor and sensory functions. MS is by nature an inflammatory disease, contributed by the combined action of many types in central and peripheral immune cells (Dendrou, Fugger, \& Friese, 2015). However, the exact neuroinflammatory processes that underpin the pathophysiology of the disease are still not fully understood. It has long been postulated that a T-cell driven inflammatory response, potentially caused by peripherally activated T helper 1 (Th1) or Th17 cells that migrate to the central nervous system (CNS), initiates a chain of events that lead to progressive demyelination and axonal damage (Legroux \& Arbour, 2015). However, an expanding body of evidence has suggested that the innate immune system is also important in initiating or maintaining inflammation. Microglia, dendritic cells, natural killer cells, and mast cells have all been implicated in the pathogenesis of MS (Gandhi, Laroni, \& Weiner, 2010). In particular, the role of microglia as potentially important innate immune cells involved in the axonal and axoglial disruption seen in chronic MS, has drawn attention to the mechanisms underlying immune cell activation in the pathophysiology of the disease (Bogie, Stinissen, \& Hendriks, 2014; Carson, 2002; Goldmann \& Prinz, 2013). Various factors can activate microglia including colony-stimulating factor-1 (CSF1) (Kierdorf \& Prinz, 2013). CSF1 is a cytokine crucial for microglial proliferation, maturation, and survival during development and in adult CNS (Elmore et al., 2014; Kondo \& Duncan, 2009). CSF1 can activate the tyrosine kinase in the intracellular domain of CSF1 receptor (CSF1R), triggering an intracellular signaling pathway (Chitu, Gokhan, Nandi, Mehler, \& Stanley, 2016). Another cytokine, interleukin-34 (IL34), has also been identified as a ligand of CSF1R and is required for the development of microglia (Wang et al., 2012). CSF1-CSF1R signaling pathway has been reported to be responsible for the proliferation of microglia in mouse models of prion 
(Gomez-Nicola, Fransen, Suzzi, \& Perry, 2013) and Alzheimer's diseases (Olmos-Alonso et al., 2016), and amyotrophic lateral sclerosis (Martinez-Muriana et al., 2016). The involvement of CSF1 in the pathogenesis of MS has also been implicated in previous studies. In a rat MS model, experimental autoimmune encephalomyelitis (EAE), CSF1 mRNA levels in the spinal cords detected by Northern blot increased significantly before the onset of early clinical signs and peaked before maximal clinical incidence of disease (Hulkower et al., 1993). Recently it was reported that CSF1 immunoreactivity in the spinal cords in the early pre-symptomatic phase of rat EAE model was increased (Borjini, Fernandez, Giardino, \& Calza, 2016). These data indicate that CSF1 upregulation may contribute to the initiation of neuroinflammation in EAE. However, there is no detailed study on the actual levels of CSF1 and IL34 proteins and the cellular expression of CSF1 proteins in the CNS of EAE mice at both acute and chronic stages. Our laboratory has developed an EAE model in Biozzi ABH mice that closely mimics the relapsing-remitting and secondary progressive MS (Al-Izki et al., 2012). Using this animal model, we measured the CSF1 and IL34 levels in the plasma and in the spinal cords, the main site of pathology of EAE mice. We also identified the cell types that express and upregulate CSF1 in acute and chronic EAE, and explored the potential relationship between activated microglia and the death of CSF1-expressing neurons. Furthermore, we examined the effects of viral vector mediated over-expression of CSF1 in spinal neurons on the proliferation and activation of microglia and the loss of the CSF1transduced neurons.

\section{MATERIALS AND METHODS}

\subsection{Induction of EAE in mice and sample collection}

Experiments were performed in accordance with the United Kingdom Animals (Scientific Procedures) Act 1986, following local ethical review of procedures. Acute and chronic mouse 
EAE models were induced according to the method used routinely in our laboratory (Al-Izki et al., 2012; Baker et al., 1990; Pryce et al., 2005). Biozzi ABH mice (both male and female, 8 weeks old) bred in our Biological Service Unit were used. Briefly, spinal cord homogenate emulsified with complete Freunds adjuvant (incomplete Freund's adjuvant supplemented with both Mycobacterium tuberculosis and M. butyricum) was injected subcutaneously in both hindflanks and again 7 days later. Mice were monitored during the relapsing-remitting phase of disease progression, as previously documented.

For measurement of CSF1 and IL34 by ELISA, acute EAE mouse spinal cord samples were obtained by sacrificing animals with clinical score 4 (complete hind limb paralysis for 2 days) via $\mathrm{CO}_{2}$ asphyxiation at day 17-18 after first inoculation. Blood was obtained by severing cardiac vessels and collected in EDTA-coated tubes which were centrifuged to extract plasma. The spinal cords were ejected using hydrostatic pressure applied to the spinal column via a 20 gauge needle. All samples were frozen immediately by immersion in liquid nitrogen and stored at $-80^{\circ} \mathrm{C}$. Chronic EAE mice were sacrificed after the development of the secondary progressive phase of disease, between days 60-90 post-induction. Spinal cords and plasma were obtained using the same procedures as described above. Spinal cords and plasma were also obtained from mice 17 days after the first complete adjuvant injection, serving as controls, in addition to normal mice.

For immunohistochemistry, animals were sacrificed at the same time points as described above. After deep anesthesia, mice were transcardially perfused with saline followed with $4 \%$ paraformaldehyde. Spinal cords were dissected and postfixed in 4\% paraformaldehyde for 1 day, then transferred to $30 \%$ sucrose in PBS for 1 day. Spinal cords were then cut into three segments and embedded in OCT compound and stored at $-80^{\circ} \mathrm{C}$ until required.

\subsection{ELISA}


Spinal cord samples from normal, adjuvant-injected, acute and chronic EAE mice were weighed and then minced using a pair of small scissors. Extraction buffer composed of $0.04 \%$ bovine serum albumin and cOmplete ${ }^{\mathrm{TM}}$ protease inhibitors (Roche Diagnostics Ltd., Burgess Hill, UK) in PBS ( $\mathrm{pH}$ 7.4) was added to each sample at $5 \mu$ per mg of sample. Tissues were homogenized by sonication on ice using a Vibra-Cell sonicator (Sonics and Materials Inc., Newtown, CT, USA) at $30 \mathrm{~W}$ for $3 \times 1 \mathrm{~min}$. Homogenates were centrifuged at $10,000 \times g$ for 20 min at $4^{\circ} \mathrm{C}$. Supernatants were collected and used immediately for ELISA. Concentrations of mouse CSF1 and IL34 in the spinal cords and plasma were measured using the Quantikine ${ }^{\circledR}$ Colorimetric Sandwich ELISA kits (R\&D Systems, Abingdon, UK) according to the manufacturer's protocols.

\subsection{Production of lentiviral vectors}

Mouse CSF1 cDNA was purchased from InvivoGen (San Diego, CA, USA). For easy identification of CSF1-transduced cells, GFP cDNA was fused in frame to CSF1 cDNA with a $2 \mathrm{~A}$ sequence (from foot-and-mouth disease virus) in-between, which allows separation of CSF1 and GFP proteins during translation by a mechanism called ribosome skipping. To make the lentiviral vector (LV) expressing CSF1-2A-GFP under the control of a neuronal specific promotor, human synapsin-1 promoter (hSyn), overlap extension PCR was used to fuse CSF1 cDNA with the 2A sequence and GFP cDNA. The fused construct was then subcloned into LV transfer vector pRRL/hSyn. LV/hSyn_CSF1-2A-GFP and the control vector, LV/hSyn_GFP, were produced based on the protocol of Dull et al.(Dull et al., 1998) using HEK293T cells as packaging cells. The harvested viral particles in the conditioned media were concentrated by ultracentrifugation, re-suspended in sterile PBS, and stored at $80^{\circ} \mathrm{C}$. 


\subsection{Immunoblotting}

To detect the secreted CSF1 in the conditioned medium, pRRL/hSyn_CSF1-2A-GFP was transfected into HEK293 cells cultured in serum-free medium (Animal-Free PeproGrow AFCHO Media, PeproTech EC Ltd, London, UK). pRRL/hSyn_GFP was also transfected as control. Two days after transfection, media were harvested and concentrated by 20 times using Amicon Ultra-15 Centrifugal Filter Units (Ultracel-10K, Millipore UK Ltd, Watford, UK). Transfected cells were washed off from the culture dish with cold PBS and spun down. Cell pellet was lysed using $400 \mu \mathrm{l}$ Cell Lysis Buffer (10 mM Tris-HCl, 1 mM EDTA, 0.3\% NP-40, cOmplete ${ }^{\mathrm{TM}}$ proteinase inhibitors (Roche)) and centrifuged at $13,000 \times g$ for $5 \mathrm{~min}$ at $4^{\circ} \mathrm{C}$. Supernatant was transferred to another tube and stored at $-80^{\circ} \mathrm{C}$ until required.

Conditioned media or cell lysates ( $9 \mu \mathrm{l}$ each) were mixed with reducing loading buffer, heated at $70^{\circ} \mathrm{C}$ for $10 \mathrm{~min}$, and loaded into a 4-12\% SDS-PAGE precast gel (Expedeon, Cambridge, UK). Proteins were transferred to PVDF membrane, which was incubated in blocking buffer for $1 \mathrm{~h}$ (10\% skimmed milk in TBST buffer (Tris buffered saline $+0.3 \%$ Tween 20). Membrane was then incubated with goat anti-murine CSF1 antibody (PeproTech, 1:1000 dilution in blocking buffer) overnight. After being washed in TBST for $3 \times 10 \mathrm{~min}$, membrane was incubated with donkey anti-goat IgG-HRP (Santa Cruz, 1:10,000 dilution in TBST). Lumigen ECL Ultra (Lumigen, Southfield, MI) was used for signal generation and ECL signal was recorded with ChemiDoc ${ }^{\mathrm{TM}} \mathrm{XRS}+$ System (BioRad Laboratories Ltd, Watford, UK).

\subsection{Intraspinal viral vector injection}

Intraspinal injection was carried out similarly to a previously published procedure (Peluffo et al., 2013). Thirty-six adult male Biozzi ABH mice (23 - 28 g) were anaesthetized with isoflurane and the third to fifth lumbar (L3-5) segments of spinal cord were exposed by a 
laminectomy. After the dura was cut open, a finely drawn glass micropipette was inserted from left L2 segment at approximately 30 degree angle to the rostral spinal cord and the tip of the pipette reached the ventral horn of L4. One $\mu \mathrm{l}$ of LV/hSyn_CSF1-2A-GFP or LV/hSyn_GFP was injected using a microinfusion pump at the speed of $200 \mathrm{nl} / \mathrm{min}$. The needle was left in situ for 2 min after injection before withdrawal to prevent leakage. A second injection was made to the L5 ventral horn in order to transduce more neurons in the lumbar enlargement. The muscle and skin incisions were then sutured. Animals were monitored daily after surgery and provided with analgesia twice daily for 2 days. A group of 6 mice was culled at one, two, or three weeks after viral vector injection and processed as described above. Lumbar spinal cords containing injection sites were removed, post-fixed, and preserved in 30\% sucrose before being embedded in OCT Compound for sectioning.

\subsection{Immunohistochemistry}

Horizontal cryostat sections (16 $\mu \mathrm{m}$ thick) of spinal cords from normal and adjuvant-injected mice, and mice with acute EAE and chronic EAE were cut, mounted onto SuperFrost Plus glass slides, and dried at room temperature for $30 \mathrm{~min}$. Sections were either used immediately or stored at $-20^{\circ} \mathrm{C}$. Most of the spinal cord sections were double-immunostained for CSF1 (goat-anti mouse CSF1) and a cellular marker for myelin, astrocytes, neurons, or microglia using rabbit antibodies (see Table 1 for a list of primary antibodies used) and the immunosignals were detected with two different fluorophore-conjugated secondary antibodies. Double immunostaining for CSF1 and a cellular marker was performed in two steps in order to avoid the cross-reactions of secondary antibodies. Incubation was carried out in a humidified chamber at room temperature and washing was done for $3 \times 10 \mathrm{~min}$ in TBST buffer $(0.1 \%$ Tween-20). For immunostaining of CSF1, sections were washed and incubated with Blocking Solution 1 (10\% donkey serum, 0.3 M glycine, 0.3\% Triton X-100 in PBS) at 
room temperature for $2 \mathrm{~h}$. Goat anti-CSF1 antibody diluted in blocking solution 1 was applied and incubated at room temperature overnight. The slides were washed and incubated with Biotin-SP AffiniPure Donkey anti-Goat IgG (H+L) (1:200, Jackson ImmunoResearch Laboratories, Inc, West Grove, PA) for $80 \mathrm{~min}$. Sections were washed again and incubated with Alexa Fluor-488 conjugated streptavidin (1:2000, Thermo Fisher) for 30 min. Sections were washed and the immunostaining for another molecule started by incubating with Blocking Solution 2 (10\% goat serum, 0.3 M glycine, 0.3\% Triton X-100 in PBS) for $2 \mathrm{~h}$. Sections were incubated with rabbit antibodies against myelin-associated glycoprotein (MAG), glial fibrillary acidic protein (GFAP), NeuN, or Iba1 diluted in Blocking Solution 2 overnight. After washing, Alexa Fluor-568 conjugated goat anti-rabbit antibody (1:2000, Thermo Fisher) was applied and incubated for $2 \mathrm{~h}$. Sections were washed in TBST first, then in water briefly. DAPI-Fluoromont-G (SouthernBiotech, Birmingham, AL, USA) were used to stain the nuclei and sections were sealed under a coverslip.

To verify the specificity of the goat anti-mouse CSF1 antibody from PeproTech, another goat anti-mouse CSF1 antibody from R\&D Systems (Table 1) which has been used for immunostaining CSF1 in DRG and spinal motoneurons (Guan et al., 2016), was tested on our spinal cord sections. Both antibodies stained neurons and astrocytes with similar pattern of immunoreactivity (data not shown).

To detect the expression of CSF1 and GFP in spinal neurons in LV/hSyn_CSF1-2A-GFP injected spinal cord, double immunostaining for CSF1 and GFP was performed on cross sections (16 $\mu \mathrm{m}$ thick) using a goat anti-mCSF1 and a rabbit anti-GFP antibodies (see Table 1). For labelling LV/hSyn_CSF1-2A-GFP or LV/hSyn_GFP transduced neurons and microglia, sections were double immunostained using a rabbit anti-GFP and a goat anti-Iba1 antibody. Furthermore, some sections from mice killed two weeks post viral vector injection were double immunostained with a sheep anti-GFP antibody and a rabbit anti-TMEM119 (a 
microglia-specific marker) antibody. For detecting demyelination in the viral vector transduced area, sections were double immunostained with a sheep anti-GFP antibody and a rabbit anti-MAG antibody. Double immunostaining with a sheep anti-GFP antibody and a rabbit anti-CD3 (a T cell marker) antibody were performed to check whether T-cells also accumulated close to the viral vector transduced neurons.

Confocal microscopy images of CSF1 and Iba1 double immunostained sections of acute EAE spinal cords were captured by a Zeiss LSM 710 confocal microscope using a $63 \times$ oil objective (Bio Imaging Centre, School of Engineering and Materials Science, Queen Mary University of London). Images were taken at a z-stack of 34 sections with section interval of $0.348 \mu \mathrm{m}$. Each series of z-stack images was processed with a maximum intensity projection tool to create a final image with pixels containing the maximum value at the whole stack.

\section{7 | Quantification and statistical analysis}

In order to quantify the increased expression of CSF1 in spinal neurons and neuronal loss in acute and chronic EAE mice, horizontal sections of the lumbar spinal cords were serially cut and double immunostained for NeuN and CSF1. From each animal four sections cut across the anterior gray matter were analyzed. One section from every three serial sections was chosen to avoid the potential double counting of the same neurons in adjacent sections. Four images from each section were photographed with a $20 \times$ objective and the image size of each photo is $696 \times 520 \mu \mathrm{m}$. Only neurons with clearly defined nuclei (by DAPI staining) were counted and the numbers of neurons from each animal (16 images) were pooled. Measurement of the areas of neuronal somata was performed using ImageJ (NIH). Neurons with areas of their somata larger than $350 \mu \mathrm{m}^{2}$ are categorized as large neurons, putatively, $\alpha$ motoneurons (Friese et al., 2009). Since there is a base-level expression of CSF1 in the 
neurons, the threshold for high-level expression of CSF1 in spinal neurons was artificially set based on the CSF1 immunoreactivity in normal spinal cord.

For quantifying the CSF1 levels at the peripheral white matter, four images from each section were photographed with a $40 \times$ objective and the integrated density of CSF1 immunoreactivity in a frame of $250 \times 180 \mu \mathrm{m}$ along the edge of the spinal cord was measured using ImageJ. Four sections per animal were measured and the data were pooled.

For quantification of LV/hSyn_CSF1-2A-GFP- and LV/hSyn_GFP-transduced large neurons in the ventral horns, one section was selected from every six consecutive sections in the viral vector transduced segments. Since the numbers of large $\mathrm{GFP}^{+}$neurons $\left(>350 \mu \mathrm{m}^{2}\right)$ with clear nuclear profiles in each section were low, we counted 30 sections in each animal and pooled the data.

To quantify the degree of demyelination in the areas transduced by viral vectors, ImageJ was used to measure the integrated densities of MAG immunoreactivities. Images were acquired with a $20 \times$ objective. A transduced area shown by GFP-immunoreactivity was drawn manually and the integrated density and the size of the area were measured. An adjacent untransduced area of similar size was drawn and the integrated density and size were measured. The integrated density per $\mu \mathrm{m}^{2}$ was used for data analysis. Eight sections with an interval of $96 \mu \mathrm{m}$ between them from each animal were measured and averaged.

Data are presented as mean $\pm \mathrm{SD}$. One-way ANOVA with post hoc Tukey test was used to analyze the ELISA data, to compare the numbers of $\mathrm{NeuN}^{+}$and $\mathrm{CSF}^{+}$neurons, and to compare the levels of CSF1 immunoreactivity in the white matter in normal and EAE mice. It was also used to analyze the differences in the numbers of large $\mathrm{GFP}^{+}$neurons in LV/hSyn_CSF1-2A-GFP-and LV/hSyn_GFP-injected mice at 1, 2 and 3 week post injection.

\section{RESULTS}




\subsection{CSF1 levels are significantly higher in the spinal cords of acute and chronic EAE mice}

ELISA data showed that in normal ABH mouse spinal cords, CSF1 was expressed to a level of $799 \pm 137 \mathrm{pg} / \mathrm{g}$ wet tissue (Figure 1a). Injection of adjuvant did not significantly change the level of CSF1 in the spinal cords. However, in the spinal cords of acute EAE mice CSF1 level was significantly higher than those in normal and the adjuvant-injected mice. Although the levels of CSF1 in the spinal cords from chronic EAE mice ranged from 886 to $1917 \mathrm{pg} / \mathrm{g}$ wet tissue among the six mice, the average level was significantly higher than the normal and the adjuvant-injected mice, but not significantly different from the acute EAE mice (Figure 1a). The CSF1 level in normal mouse plasma was $722 \pm 153 \mathrm{pg} / \mathrm{ml}$, which was similar to that observed in the spinal cord of normal mice (Figure 1a). CSF1 level in acute EAE mouse plasma was significantly lower than that in normal mice, but not significantly different from the chronic EAE mice (Figure 1a). Interestingly, CSF1 level in the plasma of the adjuvantinjected mice was significantly higher than all the other three groups.

IL34 level in normal mouse spinal cord was $7690 \pm 1054 \mathrm{pg} / \mathrm{g}$ wet tissue (Figure 1b) and adjuvant injection did not significantly change the IL34 level in the spinal cord. In the spinal cords of both acute and chronic EAE mice, IL34 levels were also not significantly different from the normal and adjuvant-injected mice. In chronic EAE mice IL34 levels in individual mice also varied significantly (Figure 1b). The IL34 levels in the plasma were much lower than in the spinal cords and they were at the lower limit for detection with the ELISA kit used.

\subsection{CSF1 expression increases significantly in the spinal cords of acute and chronic} EAE mice

Immunostaining for CSF1 showed that CSF1 was constitutively expressed in normal spinal cord (Figure 2a). Since CSF1 is secreted from cells and exists in the intercellular space as 
well as in the cells expressing it, low-level CSF1 immunoreactivity was seen throughout the spinal section. Immunostaining without primary anti-CSF1 antibody showed a much lower level of background staining (Figure S1), indicating that the CSF1 immunoreactivity was due to bound anti-CSF1 antibody. Cells associated with meninges (yellow arrow in Figure 2a) and a neuron-like cell (yellow arrowhead in Figure 2a) displayed stronger CSF1 immunoreactivity. CSF1 expression level in the nerve roots was rather low, with only a few $\mathrm{CSF}^{+}$cells visible (white arrowhead in Figure 2a,). Similar pattern of CSF1 immunoreactivity as that in normal mouse spinal cord was observed in the spinal cord of the adjuvant-injected mouse (Figure 2e). In both acute and chronic EAE mice CSF1 expression significantly increased in the spinal cords. In acute EAE spinal cords, the most predominant increase in CSF1 expression was observed in the neuron-like cells in the gray matter and astrocyte-like cells in the white matter (Figure 2i). There was also a noticeable decrease in the number of neuron-like cells with high-level CSF1 in chronic EAE spinal cord (Figure 2m) compared with the acute EAE spinal cord (Figure 2i). These findings were further studied with double immunostaining for a neuronal marker NeuN and an astrocyte marker GFAP, which are described below.

Myelin in both the spinal cord and a nerve root was identified by MAG immunoreactivity. Axons were arranged in an orderly pattern in the spinal cords of normal and adjuvant-injected mice (Figure $2 \mathrm{~b}$ and $2 \mathrm{f}$ ). In the spinal cord with acute EAE, axons showed a certain degree of disorganization (Figure $2 \mathrm{j}$ ) and small patches of demyelination in the peripheral white matter were observed (not shown). In the spinal cord with chronic EAE, some areas in the peripheral white matter displayed strong CSF1 immunoreactivity (marked by asterisks in Figure 2m), but weak MAG-immunoreactivity (marked by asterisks in Figure 2n), indicating demyelination in these areas. The cells with strong CSF1 immunoreactivity in 
these areas resembled reactive astrocytes, which was confirmed by the immunostaining for GFAP (see the results in 3.3).

\subsection{CSF1 expression in astrocytes in the white matter increases significantly in acute and chronic EAE mice}

Base-level expression of CSF1 in astrocytes in normal spinal cord was shown by double immunostaining of spinal cord sections for CSF1 and the astrocyte marker GFAP (Figure 3a d). Some astrocytes associated with meninges (glial limitans) expressed higher-level of CSF1 (pointed by white arrows in Figure 3d). In the spinal cord of acute EAE mice, GFAP expression increased in the astrocytes, especially those in the peripheral regions of the white matter, indicating the activation of astrocytes. The reactive astrocytic processes were much thicker and longer (hypertrophy) and CSF1 levels in these reactive astrocytes and their processes were significantly higher (pointed by the white arrowheads in Figure $3 \mathrm{~h}$ ). In the spinal cord of chronic EAE, patches in the peripheral white matter showed high-level CSF1 expression (Figure 3i) which overlapped with the high-level GFAP immunoreactivity, reflecting the presence of astrogliosis in these areas (Figure 3j). Higher density of cells was also shown in these areas as demonstrated by the nuclear staining (Figure 3k). Quantification of the intensities of CSF1 immunoreactivity in the white matter confirmed that CSF1 was significantly upregulated in both acute and chronic EAE spinal cords (Figure 3m).

\subsection{CSF1 expression in astrocytes in the gray matter does not increase significantly in acute and chronic EAE mice}

Marked increase in the number of astrocytes was observed in the gray matter of acute and chronic EAE spinal cords (Fig. 4f and j). However no noticeable increase in CSF1 expression was observed in the astrocytes in the gray matter. Again, significant increase of CSF1 
expression was seen in motoneuron-like cells in the acute EAE spinal cord (Figure 4e), while in the gray matter of chronic EAE mouse spinal cord, much fewer motoneuron-like cells had high-level CSF1 expression (pointed by white arrow in Figure 4i) and it seems many motoneurons had degenerated giving rise to vacuolization in the gray matter (Figure 4i, j, l).

\subsection{CSF1 expression increases significantly in spinal neurons of acute and chronic EAE mice}

To confirm the identity of the neuron-like cells with high-level CSF1 expression in previous immunostaining, spinal cord sections were double immunostained for CSF1 and the neuronal marker NeuN. In normal mice the spinal cords had a low base-level expression of CSF1 in neurons (Figure 5a). In the acute EAE spinal cord, the level of CSF1 expression increased markedly, especially in large motoneurons (Figure 5e-h). In the chronic EAE spinal cord, CSF1 level in the neurons was higher than that in normal mice but lower than that in acute EAE mice (Figure 5i). Furthermore, there was a noticeable decrease in CSF1-expressing neurons.

Quantification of $\mathrm{NeuN}^{+}$and $\mathrm{CSF}^{+}$neurons showed that the number of large motoneurons in the acute EAE spinal cords was not significantly lower than that in the normal spinal cord, but the number of large motoneurons with high-level CSF1 was significantly higher in acute EAE spinal cord $(p<0.001$, Figure 6$)$. In the chronic EAE mice, the number of large motoneurons was significantly lower than those in normal and acute EAE spinal cords, and also the number of large neurons with high-level CSF1 was much lower. This may implicate that large motoneurons with high-level CSF1 had died during the progression of EAE to the chronic stage. 


\subsection{High-level CSF1 expression in white matter correlates with high density of microglia in acute and chronic EAE spinal cords}

Double immunostaining for CSF1 and a microglial marker Iba1 showed that in the white matter of normal spinal cord, microglia were predominantly ramified and in "resting" state (Figure 7b). In the acute EAE spinal cord, a large number of amoeboid microglia were seen (Figure 7f), indicating they were activated. In the peripheral white matter where astrocytes expressed high-level of CSF1 (pointed by a yellow arrow in Figure 7e) a high density of $\mathrm{Iba}^{+}$cells were adjacently present (pointed by a green arrow in Figure $7 \mathrm{f}$ ). In the peripheral region of the white matter of chronic EAE mouse spinal cord, high-level CSF1 expression (indicated by yellow arrows in Figure 7i) also correlated with a high-density of $\mathrm{Iba}^{+}{ }^{+}$cells (indicated by green arrow in Figure 7j). These observations indicate that high-level CSF1 expression promotes local proliferation or migration of microglia. However, the regions with high-density of $\mathrm{Iba}^{+}$cells may also contain a population of infiltrating macrophages as it was reported by Ajami et al. (Ajami, Bennett, Krieger, McNagny, \& Rossi, 2011).

\subsection{Microglia surround neurons with high-level CSF1 in acute and chronic EAE spinal cord}

Similarly as in the white matter, motoneurons increased the expression of CSF1 significantly (Figure $8 \mathrm{~d}$ ) and a high-density of activated microglia was in very close proximity of CSF $1^{+}$ neurons in the gray matter of acute EAE spinal cord (Figure 8f). This can be seen more clearly using a confocal microscope (Figure $8 \mathrm{~g}-\mathrm{i}$ ). In the anterior gray matter of chronic EAE spinal cord, the number of neurons with high-level CSF1 expression was much lower (Figure $8 \mathrm{j}, \mathrm{m}, \mathrm{p}$ ). The neurons with high-level CSF1 were enwrapped by microglia (indicated by white arrows in Figure 81, o, and r). Interestingly, the morphology of microglia in the acute and chronic EAE mice was different, with more ramified microglia surrounding the 
$\mathrm{CSF}^{+}$neurons in the chronic EAE spinal cord while more amoeboid microglia in the acute EAE mice. In the spinal cord of chronic EAE mice microglia were also observed to be associated with neurites of $\mathrm{CSF}^{+}$neurons (Figure $9 \mathrm{c}$ and $\mathrm{f}$ ), demonstrating the close relationship between the level of CSF1 and the aggregation of microglia.

Double immunostaining of spinal cord sections for CSF1 and Iba1 showed that microglia did not express CSF1 in normal, acute EAE, and chronic EAE spinal cords. Orthogonal views of the Z-stack confocal images demonstrated no overlapping immunoreactivities of CSF1 and Iba1 in the acute EAE spinal cord gray matter (Figure S2).

\subsection{Lentiviral construct mediates co-expression of CSF1 and GFP in vitro and in vivo.}

HEK293 cells were able to express the CSF1-2A-GFP construct after transfection even though a neuron-specific synapsin-1 promoter was used. CSF1 immunoreactivity was found to be mainly located on the membrane while GFP accumulated in the nuclei (Figure 10a-c), indicating that most CSF1 and GFP molecules were separated during translation. Immunoblotting showed that in the conditioned media CSF1 appeared as a broad band with molecular masses of around $140-170 \mathrm{kD}$, which was significantly higher than the predicted molecular mass of CSF1 peptides (60.6 kD) (Figure 10d). CSF1 in cell lysate showed a main band of molecular mass of around $95 \mathrm{kD}$, and weaker bands of around 140 and $250 \mathrm{kD}$ were visible as well. It is known that the predominant soluble form of CSF1 is a chondroitin sulfate-containing proteoglycan (Price, Choi, Rosenberg, \& Stanley, 1992), which explains for the much larger and a broad range of molecular masses of the secreted CSF1.

Injection of LV/hSyn_CSF1-2A-GFP into mouse spinal cords led to expression of CSF1 and GFP in neuronal cells at and around the injection site. GFP fluorescence in most transduced neurons was undetectable without using an anti-GFP antibody. Expression of CSF1 and GFP in neurons at 1 week post injection was clearly detected and the 
immunoreactivity of GFP and CSF1 co-existed in the same neurons and their neurites (Figure 10e-g). Since GFP immunoreactivity represented CSF1 immunoreactivity well, for the follow-on double immunostaining of LV/hSyn_CSF1-2A-GFP-injected spinal cord sections an anti-GFP antibody was used to indicate the CSF1 levels in transduced neurons and neurites.

\subsection{Over-expression of CSF1 in spinal neurons leads to significant increase in microglial} density.

Double immunostaining for GFP and Iba1 of spinal cord sections from normal Biozzi ABH mice sacrificed at 1, 2, and 3 weeks post LV/hSyn_GFP injection showed good transduction of neurons (Figure 11a, e, i). Densities of microglia in GFP-transduced areas were similar to those adjacent untransduced areas. Densities of microglia did not change at 1, 2, and 3 weeks post injection (Figure 11b, f, j). The microglia in the transduced areas were ramified, implicating that they were in the "resting" state. These results showed that GFP expression in neurons did not grossly activated microglia. On the other hand, injection of LV/hSyn_CSF12A-GFP induced marked increase in microglial densities in CSF1-GFP-transduced areas 1, 2, and 3 weeks post injection (Figure $11 \mathrm{n}, \mathrm{r}, \mathrm{v}$ ). The morphology of the microglia indicates that they were activated, especially those 1 week post injection (Figure 11n). CSF1-GFP ${ }^{+}$neurons were enwrapped by microglia (Figure $11 \mathrm{p}, \mathrm{t}, \mathrm{x}$ ), similar to that seen in chronic EAE mouse spinal cords (Figure 8j, o, r). Apart from enwrapping CSF1-GFP ${ }^{+}$neuronal somata, microglia were closely associated with the $\mathrm{CSF}^{+}$neurites as well (Figure $13 \mathrm{e}-\mathrm{h}$ and $\mathrm{i}-\mathrm{l}$ ), similar to what is shown in chronic EAE spinal cord (Figure 9). Microglia were not seen to be associated with $\mathrm{GFP}^{+}$neurites of LV/hSyn-GFP transduced neurons (Figure 13a-d)

Clusters of $\mathrm{Iba}^{+}$cells were seen in the CSF1-GFP transduced areas, which were more prominent 2 week post injection (marked by * in Figure $11 \mathrm{r}$ ). Those $\mathrm{Iba}^{+}$cells in clusters 
were not macrophages penetrated into the parenchyma of spinal cord as these cells were also positive for a new microglia-specific marker, TMEM119 (Bennett et al., 2016), confirming that they were indeed microglia (Figure 3S).

\subsection{Over-expression of CSF1 in spinal neurons leads to the loss of large transduced} neurons.

We noticed that there were fewer large CSF1-GFP-transduced neurons in ventral horn (presumably motoneurons) at 2 and 3 weeks post injection than that at 1 week (Figure $11 \mathrm{~m}, \mathrm{q}$, u), therefore, we counted the GFP ${ }^{+}$large neurons in both LV/hSyn_GFP and LV/hSyn_CSF1-2A-GFP injected groups. In LV/hSyn_GFP injected group, there was no difference in the number of large $\mathrm{GFP}^{+}$neurons at 1, 2 and 3 weeks, however, in LV/hSyn_CSF1-2A-GFP injected group the numbers of large $\mathrm{GFP}^{+}$neurons were significantly lower than that at 1 week (Figure 12), suggesting that CSF1 induced proliferation and activation of microglia seen in Figure 11 led to the loss of the large neurons.

\subsection{Over-expression of CSF1 in spinal neurons causes demyelination of CSF1-} expressing neurons.

To examine whether the proliferation and activation of microglia induced by over-expressed CSF1 led to demyelination as microglia were closely associated with $\mathrm{CSF}^{+}$neurites (Figure 13h, 1), the myelin levels in the spinal sections immunostained with MAG were studied. As expected, there was no obvious difference in the levels of MAG immunoreactivity in the GFP-transduced and untransduced areas (Figure 14b). However, there was noticeable reduced level of MAG immunoreactivity in the CSF1-GFP transduced areas (marked by * in Figure 14f) compared with the adjacent untransduced areas. The level of MAG immunoreactivity in CSF1-GFP transduced area was $46 \%$ lower than that in the adjacent untransduced area 
(Figure 14i). We further examined the sections using confocal microscopy and found that the $\mathrm{GFP}^{+}$large axons (about $5 \mu \mathrm{m}$ in diameter) of GFP transduced neurons were smooth and myelinated as GFP and MAG immunoreactivities were closely associated (pointed by white arrows in Figure 141 and o). However, the CSF1-GFP ${ }^{+}$axons showed limited association with MAG immunoreactivity (Figure 14r and $\mathrm{u}$ ).

\section{DISCUSSION}

In this study we showed that CSF1 levels were markedly elevated in acute EAE mouse spinal cord. The elevated CSF1 levels in acute EAE spinal cords were similar within the treatment group, which may reflect the uniformity of the onset of the initial attack in the mice after induction (Al-Izki et al., 2012). The CSF1 levels in chronic EAE mouse spinal cords were also elevated, however, the levels varied significantly within the group, which may be due to the big variation in severity of neurodegeneration in individual mice at chronic stage of disease (Al-Izki et al., 2012). Our results on elevated CSF1 protein levels in spinal cords agree with a study on mRNA levels in rat spinal cords with acute EAE (Hulkower et al., 1993). By measuring the densities of the mRNA bands in Northern blots CSF1 mRNA levels in rat spinal cords was shown to increase 8 days after induction and reached peak levels on day 12 , the height of clinical symptoms. Using a colony stimulating assay as an indirect measurement for the levels of CSF1 proteins in the rat spinal cord, they also found that spinal cord homogenates from rats 12 days post induction had the highest activity in stimulating bone marrow cell colony formation.

The other CSF1R ligand IL34 in mouse spinal cord has a higher base-level than CSF1. However, we found that IL34 levels did not change significantly in the spinal cords of acute and chronic EAE mice, implicating that CSF1 is the key cytokine in inducing proliferation and activation of microglia in response to challenge of spinal cord homogenates. Similarly, in 
an ALS model, CSF1 mRNA and proteins increased in spinal cords, but with no change in IL34 mRNA level (Martinez-Muriana et al., 2016). In a peripheral nerve injury model, CSF1 mRNA levels in dorsal root ganglia (DRG) and spinal cords increased significantly while IL34 mRNA levels did not change (Okubo et al., 2016). However, in a prion disease model, using in situ hybridization, it was shown that CSF1, IL34, and CSF1R mRNAs increased in the hippocampus and thalamus (Gomez-Nicola et al., 2013). These results imply that IL34 may respond differently in different pathological conditions.

In our study CSF1 concentrations in blood plasma decreased in acute EAE mice, but the reduction was not significant in chronic EAE mice, indicating that the elevated CSF1 in the spinal cords of EAE mice was produced in situ. In a recent study, it was reported that CSF1 concentration in rat plasma increased one day after EAE induction, but then continued decreasing into the initial acute attack (Borjini et al., 2016). The mechanism underlying such change of plasma CSF1 remains to be determined. CSF1 level in plasma of the adjuvantinjected mice was significantly higher than that of normal mice, which may reflect the peripheral inflammation induced by adjuvant. It is also higher than the mice with chronic EAE, which may be explained by that adjuvant-induced inflammation had subsided 60-90 days post induction. However, a reason for the lower CSF1 level in plasma in acute EAE mice in comparison with the normal and the adjuvant-injected mice remains unclear.

The increased expression of CSF1 in the acute and chronic EAE mouse spinal cord was supported by immunohistochemical studies. Constitutive expression of CSF1 was detected in normal mouse spinal cords and the main types of cells that express CSF1 are astrocytes and neurons. Our results support the findings in some previous studies reporting the presence of mRNAs for CSF1 in astrocytes and certain types of neurons in the brain (Calvo, Dobbertin, Gelman, Glowinski, \& Mallat, 1998; Frei, Nohava, Malipiero, Schwerdel, \& Fontana, 1992; Nohava, Malipiero, Frei, \& Fontana, 1992). Astrocytes associated with pia mater expressed a 
higher level of CSF1 than those inside the spinal cord parenchyma. In acute EAE mice, astrocytes were activated, especially in the peripheral region of the white matter, and CSF1 expression was markedly increased in these reactive astrocytes. A base-level expression of CSF1 is essential for maintaining a stable pool of microglia in the CNS of normal mice. However, increased CSF1 expression in the peripheral white matter also correlated with higher density of $\mathrm{Iba}^{+}$cells in the same regions, where major pathological changes occur in EAE. We postulate that the increased expression and secretion of CSF1 in those astrocytes stimulate proliferation and activation of microglia, which may release inflammatory factors and participate in the demyelination process. Astrocytes have been regarded as an important player in various CNS pathological conditions including MS and they may actively participate in both lesion development and repair (Correale \& Farez, 2015). Recently it was reported that a type of neurotoxic reactive astrocytes were induced by activated microglia, which results in the death of neurons and oligodendrocytes (Liddelow et al., 2017). Therefore, microglia and astrocytes can interact with each other and exuberate demyelination and neurodegeneration.

Large motoneurons in the spinal cords also significantly increased the expression of CSF1 in acute and chronic EAE, which may be responsible for increased proliferation and activation of microglia in the gray matter. Neuronal loss was not so obvious in the acute stage, but significant loss of large motoneurons was observed in the chronic stage. Since many large motoneurons with high-level CSF1 expression were encircled by microglia in the spinal cords from both acute and chronic EAE mice, we presume that neuronal loss may be partly due to the action of microglia. A study on a mouse ALS model supports our presumption, in that CSF1 is found to be responsible for microgliosis and increased neuronal death, and treatment with GW2580, a CSF1R inhibitor, slowed disease progression, attenuated motoneuron death, and extended the survival of the mice (Martinez-Muriana et al., 2016). Similarly, depletion of 
microglia from Alzheimer's mice by oral administration of CSF1R inhibitor PLX3397 prevented neuronal loss and rescued dendritic spine loss, further implicating that microglia contribute to neuronal loss in neurodegenerative disorders (Spangenberg et al., 2016). Studies using two other CSF1R inhibitors, PLX5622 and GW2850, also demonstrated improved learning and memory in mouse models of Alzheimer's disease (Dagher et al., 2015; OlmosAlonso et al., 2016). Inhibition of CSF1R with GW2850 in a prion disease model led to significant reduction of neuronal degeneration and improvement in the behavioral performance and survival (Gomez-Nicola et al., 2013). Furthermore, in a study with the intention to exploit the neuroprotective effect of CSF1, the researchers found that administration of CSF1 to an ALS mouse model accelerated disease progression and shortened survival of the animals (Gowing, Lalancette-Hebert, Audet, Dequen, \& Julien, 2009). The CSF1-treated animals had increased number of microglia and upregulated proinflammatory cytokines TNF- $\alpha$ and IL-1 $\beta$. These studies support the notion that CSF1CSF1R signaling pathway promotes inflammation and may play a key role in the progression of neurodegeneration. However, some studies suggest that CSF1-CSF1R signaling pathway is neuroprotective. Luo et al. showed that administration of CSF1 or IL34 prevented the loss of pyramidal neurons and microgliosis in mouse hippocampus after kainic acid injection (Luo et al., 2013). Furthermore, they also found that systemic administration of CSF1 ameliorated memory deficits in a transgenic mouse model of Alzheimer's disease, which contradicts the findings from other research groups (Dagher et al., 2015; Olmos-Alonso et al., 2016; Spangenberg et al., 2016). In another hippocampal lesion model using induced expression of diphtheria toxin A-chain, Rice et al. found that eliminating microglia using PLX3397 improved functional outcomes after lesioning, but potentiated neuronal loss during lesion formation (Rice et al., 2015). In a brain ischemia model, Jin et al. reported that depletion of microglia by blocking CSF1R using PLX3397 exacerbated neurological deficits and brain 
infarction (Jin et al., 2017). However, Li et al. reported that eliminating microglia with PLX3397 attenuated brain injury after intracerebral hemorrhage (Li et al., 2017). Although cytotoxicity of microglia has been recognized for a long time (Banati, Gehrmann, Schubert, \& Kreutzberg, 1993), latest studies indicate that microglia may exert different effects in different types of lesions and at different stages of the lesions. It is generally recognized that microglia exist in different phenotypes with different functions (Boche, Perry, \& Nicoll, 2013) and whether they are neuroprotective or neurotoxic may depend on the pathological conditions and/or age of the brain and spinal cord (Biber, Owens, \& Boddeke, 2014).

We also observed that microglia were in close contact with $\mathrm{CSF}^{+}$axons, which raises the possibility that those microglia might also damage the axons and cause demyelination. Clusters of activated microglia were observed in the white matter of brain from MS patients (Singh et al., 2013; van Horssen et al., 2012). However, it is unknown whether those clusters were induced by local increase of CSF1. Microglia nodules were reported to be associated with degenerating axons (Singh et al., 2013). We did find that in the peripheral regions of the spinal cord white matter, demyelinated areas displayed high-level CSF1 immunoreactivity and accumulation of $\mathrm{Iba}^{+}$cells, presumably microglia and the infiltrated macrophages. We postulate that initial damage by adaptive immune cells on CNS myelin activates astrocytes which increase the expression and release of CSF1, which stimulates proliferation and activation of microglia and further exuberates axonal damage. A line of evidence to support the link between CSF1 and demyelination comes from a study on Charcot-Marie-Tooth neuropathy (Groh et al., 2015; Groh et al., 2012). By crossbreeding mice suffering from Charcot-Marie-Tooth neuropathy with mice carrying a spontaneous null mutant for CSF1, it was found that the cross-bred mice demonstrated a robust and persistent amelioration of demyelination and axon perturbation in the peripheral nerves. In the biopsies from patients with Charcot-Marie-Tooth type 1, frequent cell-cell contacts between macrophages and 
CSF1-expressing endoneurial fibroblasts were also observed, similar to what we observed in EAE spinal cords, where microglia were in close contact with CSF1-expressing neurons and axons.

It has been known that microglia activated by inflammation can phagocytose stressed but viable neurons, resulting in neuronal death by phagoptosis, cell death caused by phagocytosis of the cells (Brown \& Neher, 2014). An in vitro experiment showed that CSF1 stimulated phagocytic activity of adult human microglia (Smith et al., 2013). It was previously observed that injury to a peripheral nerve led to proliferation and hypertrophy of microglia around the axotomized motoneurons and in the central projection territories of the peripherally axotomized sensory neurons, where they phagocytosed degenerating axons and terminals (Svensson et al., 1993). Recently the signal molecule for such microglial reaction to peripheral nerve injury was identified as CSF1 (Guan et al., 2016). Increased secretion of CSF1 may act as an injury signal and elicit inflammatory reaction, therefore, we postulate that in EAE immune attack on CNS myelin triggers the upregulation of CSF1 and induces the proliferation, activation, and phagocytosis of microglia, which in turn, damage and phagocytose the stressed neurons and the axons.

In order to confirm that increased CSF1 does act as an injury signal, we expressed CSF1 in spinal neurons to mimic the increased expression of CSF1 in these neurons in EAE mice. Indeed, over-expression of CSF1 in neurons led to profound proliferation and activation of microglia in the transduced areas, formation of microglial clusters, and enwrapping of microglia of CSF1-expressing neurons and axons, similar to what have been observed in the EAE spinal cords. The loss of CSF1-transduced large motoneurons occurred much faster than that in EAE spinal cords, which may be due to significantly larger amount of CSF1 was secreted by the transduced neurons than those neurons in EAE spinal cords, inducing stronger response from microglia. Such explanation is supported by the differences in microglial 
densities in the gray matter of acute EAE spinal cord and in CSF1-transduced areas. However, neuronal loss in EAE may be due to a combination of various factors apart from microgliosis induced by increased expression CSF1. Motoneurons in EAE may have been injured by other types of cells such as T-cells. Activated microglia act to remove the injured neurons. T-cells were seen accumulated along the needle tracks in both LV/Syn_CSF1-2A-GFP and LV/Syn_GFP injected spinal cords. However, in the viral vector transduced areas away from the needle tracks, the number of T-cells was relatively low and not seen to be close to CSF1or GFP-transduced neurons (Figure S4), which implicates that T-cells may not be directly involved in the loss of large motoneurons with induced high-level CSF1 expression.

Over-expressing CSF1 in neurons also led to the demyelination of the transduced neurons, which is not surprising since large numbers of activated microglia accumulated in the transduced areas. Close contact of microglia with $\mathrm{CSF} 1^{+}$axons of CSF1-transduced neurons also mirrors what was observed in EAE spinal cords.

In summary, we have found that CSF1 was significantly upregulated in the spinal cords of both acute and chronic EAE mice. Astrocytes and neurons were identified as the main source of CSF1, which led proliferation and activation the microglia. Activated microglia were closely associated with CSF1-expressing neurons and astrocytes, and may be responsible for neuronal loss and demyelination at the chronic stage of EAE. Engineered over-expression of CSF1 in spinal neurons also led to the loss of large neurons and demyelination, mirroring what was observed in EAE spinal cords. The findings from this study support for a role of CSF1 in the pathogenesis and progression of EAE, and potentially MS, and CSF1-CSF1R signaling pathway may be a potential target for development of treatments to slow or halt the neurodegeneration in progressive MS.

\section{References}


Ajami, B., Bennett, J. L., Krieger, C., McNagny, K. M., \& Rossi, F. M. (2011). Infiltrating monocytes trigger EAE progression, but do not contribute to the resident microglia pool. Nat Neurosci, 14, 1142-1149. doi:10.1038/nn.2887

Al-Izki, S., Pryce, G., O'Neill, J. K., Butter, C., Giovannoni, G., Amor, S., \& Baker, D. (2012). Practical guide to the induction of relapsing progressive experimental autoimmune encephalomyelitis in the Biozzi ABH mouse. Mult Scler Relat Disord, 1, 29-38. doi:10.1016/j.msard.2011.09.001

Baker, D., O'Neill, J. K., Gschmeissner, S. E., Wilcox, C. E., Butter, C., \& Turk, J. L. (1990). Induction of chronic relapsing experimental allergic encephalomyelitis in Biozzi mice. $J$ Neuroimmunol, 28, 261-270.

Banati, R. B., Gehrmann, J., Schubert, P., \& Kreutzberg, G. W. (1993). Cytotoxicity of microglia. Glia, 7, 111-118. doi:10.1002/glia.440070117

Bennett, M. L., Bennett, F. C., Liddelow, S. A., Ajami, B., Zamanian, J. L., Fernhoff, N. B., ... Barres, B. A. (2016). New tools for studying microglia in the mouse and human CNS. Proc Natl Acad Sci U S A, 113, E1738-1746. doi:10.1073/pnas.1525528113

Biber, K., Owens, T., \& Boddeke, E. (2014). What is microglia neurotoxicity (Not)? Glia, 62(6), 841-854. doi:10.1002/glia.22654

Boche, D., Perry, V. H., \& Nicoll, J. A. (2013). Review: activation patterns of microglia and their identification in the human brain. Neuropathol Appl Neurobiol, 39, 3-18. doi:10.1111/nan.12011

Bogie, J. F., Stinissen, P., \& Hendriks, J. J. (2014). Macrophage subsets and microglia in multiple sclerosis. Acta Neuropathol, 128, 191-213. doi:10.1007/s00401-014-1310-2

Borjini, N., Fernandez, M., Giardino, L., \& Calza, L. (2016). Cytokine and chemokine alterations in tissue, CSF, and plasma in early presymptomatic phase of experimental 
allergic encephalomyelitis (EAE), in a rat model of multiple sclerosis. $J$

Neuroinflammation, 13, 291. doi:10.1186/s12974-016-0757-6

Brown, G. C., \& Neher, J. J. (2014). Microglial phagocytosis of live neurons. Nat Rev Neurosci, 15, 209-216. doi:10.1038/nrn3710

Calvo, C. F., Dobbertin, A., Gelman, M., Glowinski, J., \& Mallat, M. (1998). Identification of CSF-1 as a brain macrophage migratory activity produced by astrocytes. Glia, 24, 180-186.

Carson, M. J. (2002). Microglia as liaisons between the immune and central nervous systems: functional implications for multiple sclerosis. Glia, 40, 218-231. doi:10.1002/glia.10145

Chitu, V., Gokhan, S., Nandi, S., Mehler, M. F., \& Stanley, E. R. (2016). Emerging Roles for CSF-1 Receptor and its Ligands in the Nervous System. Trends Neurosci, 39, 378-393. doi:10.1016/j.tins.2016.03.005

Correale, J., \& Farez, M. F. (2015). The Role of Astrocytes in Multiple Sclerosis Progression. Front Neurol, 6, 180. doi:10.3389/fneur.2015.00180

Dagher, N. N., Najafi, A. R., Kayala, K. M., Elmore, M. R., White, T. E., Medeiros, R., ... Green, K. N. (2015). Colony-stimulating factor 1 receptor inhibition prevents microglial plaque association and improves cognition in 3xTg-AD mice. J Neuroinflammation, 12 , 139. doi:10.1186/s12974-015-0366-9

Dendrou, C. A., Fugger, L., \& Friese, M. A. (2015). Immunopathology of multiple sclerosis. Nat Rev Immunol, 15, 545-558. doi:10.1038/nri3871

Dull, T., Zufferey, R., Kelly, M., Mandel, R. J., Nguyen, M., Trono, D., \& Naldini, L. (1998). A third-generation lentivirus vector with a conditional packaging system. J Virol, 72, 8463-8471.

Elmore, M. R., Najafi, A. R., Koike, M. A., Dagher, N. N., Spangenberg, E. E., Rice, R. A., ... Green, K. N. (2014). Colony-stimulating factor 1 receptor signaling is necessary 
for microglia viability, unmasking a microglia progenitor cell in the adult brain. Neuron, 82, 380-397. doi:10.1016/j.neuron.2014.02.040

Frei, K., Nohava, K., Malipiero, U. V., Schwerdel, C., \& Fontana, A. (1992). Production of macrophage colony-stimulating factor by astrocytes and brain macrophages. $J$ Neuroimmunol, 40, 189-195.

Friese, A., Kaltschmidt, J. A., Ladle, D. R., Sigrist, M., Jessell, T. M., \& Arber, S. (2009). Gamma and alpha motor neurons distinguished by expression of transcription factor Err3. Proc Natl Acad Sci U S A, 106, 13588-13593. doi:10.1073/pnas.0906809106

Gandhi, R., Laroni, A., \& Weiner, H. L. (2010). Role of the innate immune system in the pathogenesis of multiple sclerosis. J Neuroimmunol, 221, 7-14. doi:10.1016/j.jneuroim.2009.10.015

Goldmann, T., \& Prinz, M. (2013). Role of microglia in CNS autoimmunity. Clin Dev Immunol, 2013, 208093. doi:10.1155/2013/208093

Gomez-Nicola, D., Fransen, N. L., Suzzi, S., \& Perry, V. H. (2013). Regulation of microglial proliferation during chronic neurodegeneration. J Neurosci, 33, 2481-2493. doi:33/6/2481 [pii]

10.1523/JNEUROSCI.4440-12.2013

Gowing, G., Lalancette-Hebert, M., Audet, J. N., Dequen, F., \& Julien, J. P. (2009). Macrophage colony stimulating factor (M-CSF) exacerbates ALS disease in a mouse model through altered responses of microglia expressing mutant superoxide dismutase. Exp Neurol, 220, 267-275. doi:10.1016/j.expneurol.2009.08.021

Groh, J., Klein, I., Hollmann, C., Wettmarshausen, J., Klein, D., \& Martini, R. (2015). CSF1-activated macrophages are target-directed and essential mediators of Schwann cell dedifferentiation and dysfunction in Cx32-deficient mice. Glia, 63, 977-986. doi:10.1002/glia.22796 
Groh, J., Weis, J., Zieger, H., Stanley, E. R., Heuer, H., \& Martini, R. (2012). Colonystimulating factor-1 mediates macrophage-related neural damage in a model for CharcotMarie-Tooth disease type 1X. Brain, 135, 88-104. doi:10.1093/brain/awr283

Guan, Z., Kuhn, J. A., Wang, X., Colquitt, B., Solorzano, C., Vaman, S., . . Basbaum, A. I. (2016). Injured sensory neuron-derived CSF1 induces microglial proliferation and DAP12-dependent pain. Nat Neurosci, 19, 94-101. doi:10.1038/nn.4189

Hulkower, K., Brosnan, C. F., Aquino, D. A., Cammer, W., Kulshrestha, S., Guida, M. P., . . . Berman, J. W. (1993). Expression of CSF-1, c-fms, and MCP-1 in the central nervous system of rats with experimental allergic encephalomyelitis. J Immunol, 150, 2525-2533.

Jin, W. N., Shi, S. X., Li, Z., Li, M., Wood, K., Gonzales, R. J., \& Liu, Q. (2017). Depletion of microglia exacerbates postischemic inflammation and brain injury. J Cereb Blood Flow Metab, 37, 2224-2236. doi:10.1177/0271678X17694185

Kierdorf, K., \& Prinz, M. (2013). Factors regulating microglia activation. Front Cell Neurosci, 7, 44. doi:10.3389/fncel.2013.00044

Kondo, Y., \& Duncan, I. D. (2009). Selective reduction in microglia density and function in the white matter of colony-stimulating factor-1-deficient mice. J Neurosci Res, 87, 26862695. doi:10.1002/jnr.22096

Legroux, L., \& Arbour, N. (2015). Multiple Sclerosis and T Lymphocytes: An Entangled Story. J Neuroimmune Pharmacol, 10, 528-546. doi:10.1007/s11481-015-9614-0

Li, M., Li, Z., Ren, H., Jin, W. N., Wood, K., Liu, Q., . . Shi, F. D. (2017). Colony stimulating factor 1 receptor inhibition eliminates microglia and attenuates brain injury after intracerebral hemorrhage. J Cereb Blood Flow Metab, 37, 2383-2395. doi:10.1177/0271678X16666551 
Liddelow, S. A., Guttenplan, K. A., Clarke, L. E., Bennett, F. C., Bohlen, C. J., Schirmer, L., . . Barres, B. A. (2017). Neurotoxic reactive astrocytes are induced by activated microglia. Nature, 541, 481-487. doi:10.1038/nature21029

Luo, J., Elwood, F., Britschgi, M., Villeda, S., Zhang, H., Ding, Z., . . Wyss-Coray, T. (2013). Colony-stimulating factor 1 receptor (CSF1R) signaling in injured neurons facilitates protection and survival. $J$ Exp Med, 210, 157-172. doi:10.1084/jem.20120412

Martinez-Muriana, A., Mancuso, R., Francos-Quijorna, I., Olmos-Alonso, A., Osta, R., Perry, V. H., . . Lopez-Vales, R. (2016). CSF1R blockade slows the progression of amyotrophic lateral sclerosis by reducing microgliosis and invasion of macrophages into peripheral nerves. Sci Rep, 6, 25663. doi:10.1038/srep25663

Nohava, K., Malipiero, U., Frei, K., \& Fontana, A. (1992). Neurons and neuroblastoma as a source of macrophage colony-stimulating factor. Eur J Immunol, 22, 2539-2545. doi:10.1002/eji.1830221012

Okubo, M., Yamanaka, H., Kobayashi, K., Dai, Y., Kanda, H., Yagi, H., \& Noguchi, K. (2016). Macrophage-Colony Stimulating Factor Derived from Injured Primary Afferent Induces Proliferation of Spinal Microglia and Neuropathic Pain in Rats. PLoS One, 11, e0153375. doi:10.1371/journal.pone.0153375

Olmos-Alonso, A., Schetters, S. T., Sri, S., Askew, K., Mancuso, R., Vargas-Caballero, M., .. . Gomez-Nicola, D. (2016). Pharmacological targeting of CSF1R inhibits microglial proliferation and prevents the progression of Alzheimer's-like pathology. Brain, 139, 891-907. doi:10.1093/brain/awv379

Peluffo, H., Foster, E., Ahmed, S. G., Lago, N., Hutson, T. H., Moon, L., . . Y Yanez-Munoz, R. J. (2013). Efficient gene expression from integration-deficient lentiviral vectors in the spinal cord. Gene Ther, 20, 645-657. doi:10.1038/gt.2012.78 
Price, L. K., Choi, H. U., Rosenberg, L., \& Stanley, E. R. (1992). The predominant form of secreted colony stimulating factor-1 is a proteoglycan. J Biol Chem, 267, 2190-2199.

Pryce, G., O'Neill, J. K., Croxford, J. L., Amor, S., Hankey, D. J., East, E., . . Baker, D. (2005). Autoimmune tolerance eliminates relapses but fails to halt progression in a model of multiple sclerosis. J Neuroimmunol, 165, 41-52.

doi:10.1016/j.jneuroim.2005.04.009

Rice, R. A., Spangenberg, E. E., Yamate-Morgan, H., Lee, R. J., Arora, R. P., Hernandez, M. X., . . Green, K. N. (2015). Elimination of Microglia Improves Functional Outcomes Following Extensive Neuronal Loss in the Hippocampus. J Neurosci, 35, 9977-9989. doi:10.1523/JNEUROSCI.0336-15.2015

Singh, S., Metz, I., Amor, S., van der Valk, P., Stadelmann, C., \& Bruck, W. (2013). Microglial nodules in early multiple sclerosis white matter are associated with degenerating axons. Acta Neuropathol, 125, 595-608. doi:10.1007/s00401-013-1082-0

Smith, A. M., Gibbons, H. M., Oldfield, R. L., Bergin, P. M., Mee, E. W., Curtis, M. A., . . Dragunow, M. (2013). M-CSF increases proliferation and phagocytosis while modulating receptor and transcription factor expression in adult human microglia. $J$ Neuroinflammation, 10, 85. doi:10.1186/1742-2094-10-85

Spangenberg, E. E., Lee, R. J., Najafi, A. R., Rice, R. A., Elmore, M. R., Blurton-Jones, M., . . Green, K. N. (2016). Eliminating microglia in Alzheimer's mice prevents neuronal loss without modulating amyloid-beta pathology. Brain, 139, 1265-1281. doi:10.1093/brain/aww016

Svensson, M., Eriksson, P., Persson, J. K., Molander, C., Arvidsson, J., \& Aldskogius, H. (1993). The response of central glia to peripheral nerve injury. Brain Res Bull, 30, 499506. 
van Horssen, J., Singh, S., van der Pol, S., Kipp, M., Lim, J. L., Peferoen, L., . . Amor, S. (2012). Clusters of activated microglia in normal-appearing white matter show signs of innate immune activation. J Neuroinflammation, 9, 156. doi:10.1186/1742-2094-9-156

Wang, Y., Szretter, K. J., Vermi, W., Gilfillan, S., Rossini, C., Cella, M., . . Colonna, M. (2012). IL-34 is a tissue-restricted ligand of CSF1R required for the development of Langerhans cells and microglia. Nat Immunol, 13, 753-760. doi:10.1038/ni.2360 


\section{Figure legends}

FIGURE 1 Protein levels of CSF1 and IL34 in mouse spinal cords and plasma. CSF1 and IL34 levels in spinal cord and plasma from normal and adjuvant-injected mice, and mice with acute and chronic EAE were measured by ELISA. (a) Levels of CSF1; (b) levels of IL34. Levels of CSF1 and IL34 in the spinal cord are presented as pg/g wet tissue, and $\mathrm{pg} / \mathrm{ml}$ in plasma. Values are means $\pm \mathrm{SD} . * p<0.05, * * p<0.01, * * * p<0.001$, one-way ANOVA with post-hoc Tukey test, $\mathrm{n}=6$.

FIGURE 2 CSF1 expression increases in the spinal cords of acute and chronic EAE. Double immunostaining of horizontal spinal cord sections showing the expression of CSF1 and MAG (myelin associated glycoprotein, a myelin marker) in normal (a-d), adjuvantinjected mouse (e-h), acute EAE (i-1), and chronic EAE (m-p) mice. Yellow arrow in (a) points to cells associated with meninges with high-level CSF1 expression. Yellow arrowhead in (a) point to a neuron-like cell with high-level CSF1 expression. White arrowhead in (a) points to a cell with high-level CSF1 in a nerve root. Asterisks in (m) mark the areas with high-level CSF1 in the peripheral region of the white matter. Asterisks in (n) mark the demyelinated areas with high-level CSF1. Scale bar in $(a)=200 \mu \mathrm{m}$, also applies to the other panels. GM, gray matter; WM: white matter; NR, nerve root.

FIGURE 3 CSF1 expression in astrocytes in the white matter increases significantly in acute and chronic EAE mice. Double immunostaining of horizontal spinal cord sections showing the CSF1 expression and GFAP-labeled astrocytes in normal (a-d), acute EAE (e-h), and chronic EAE (i-1) mice. Scale bar in (a) $=50 \mu \mathrm{m}$, also applies to the other panels. (m) Quantification of CSF1 immunoreactivity in the peripheral white matter. Values are means \pm SD. ${ }^{* * *} p<0.001$, one-way ANOVA with post-hoc Tukey test, $\mathrm{n}=6$. 
FIGURE 4 CSF1 expression in astrocytes in the gray matter does not increase significantly in acute and chronic EAE mice. Double immunostaining of horizontal spinal cord sections showing CSF1 expression of and GFAP-labeled astrocytes in normal (a-d), acute EAE (e-h), and chronic EAE (i-1) mice. White arrow in (i) points to motoneuron-like cells with highlevel CSF1 expression. Scale bar in (a) $=100 \mu \mathrm{m}$, also applies to the other panels.

FIGURE 5 CSF1 expression increases significantly in spinal neurons of acute and chronic EAE mice. Double immunostaining of horizontal spinal cord sections showing CSF1 expression and NeuN-labeled neurons in normal (a-d), acute EAE (e-h), and chronic EAE (i-1) mice. Scale bar in $(\mathrm{a})=100 \mu \mathrm{m}$, also applies to the other panels.

FIGURE 6 Quantification of the number of large spinal motoneurons and the number of large motoneurons with high-level CSF1 expression. Horizontal spinal cord sections from normal, acute EAE, or chronic EAE mice were double immunostained for NeuN and CSF1. Large neurons (areas of somota $>350 \mu \mathrm{m}^{2}$ ) with DAPI-stained nuclei in the anterior gray column were counted. Large neurons with high-level CSF1 expression $\left(\mathrm{NeuN}^{+} \mathrm{CSF}^{+}\right)$were also counted. Values are means $\pm \mathrm{SD} .{ }^{* * *} p<0.001$, one-way ANOVA with post-hoc Tukey test, $\mathrm{n}=6$.

FIGURE 7 High-level CSF1 expression correlates with high densities of microglia in acute and chronic EAE mice. Double immunostaining of horizontal spinal cord sections showing CSF1 expression and $\mathrm{Iba}^{+}{ }^{+}$microglia in in normal (a-d), acute EAE (e-h), and chronic EAE (i-1) mice. Yellow arrows in (e, i) point to areas with high-level CSF1 expression. Green 
arrows in (f, j) point to the corresponding areas indicated by the yellow arrows showing high densities of $\mathrm{Iba}^{+}$microglia. Scale bar in (a) $=50 \mu \mathrm{m}$, also applies to the other panels.

FIGURE 8 Microglia surround the neurons with high-level CSF1 expression in the spinal cords of acute and chronic EAE mice. Double immunostaining of horizontal spinal cord sections showing CSF1 expression and $\mathrm{Iba}^{+}$microglia in normal (a-c), acute EAE (d-i), and chronic EAE (j-r) mice. (g-i) Images of spinal cord with acute EAE acquired with a confocal microscope. White arrows in $(1, \mathrm{o}, \mathrm{r})$ point to motoneuron-like cells with high-level CSF1 expression that were enwrapped by microglia. Scale bar in $(a)=50 \mu \mathrm{m}$, also applies to (b-f) and (j-r). Scale bars in $(\mathrm{g})=10 \mu \mathrm{m}$, also applies to (h) and (i).

FIGURE 9 Microglia are closely associated with $\mathrm{CSF}^{+}{ }^{+}$neurites in the spinal cord of chronic EAE mice. Double immunostaining for CSF1 and Iba1 showed that microglia closely followed the courses of $\mathrm{CSF}^{+}{ }^{+}$neurites (pointed by white arrowheads in (c and f)). Scale bar in (a) $=25 \mu \mathrm{m}$, also applies to the other panels.

FIGURE 10 Engineered over-expression of CSF1 in vitro and in vivo. (a-c) Immunostaining of HEK293 cells transfected with pRRL/hSyn_CSF1-2A-GFP using an antimCSF1 antibody showed the GFP fluorescence was mainly located in the nuclei while CSF1 immunoreactivity was mainly located on the membrane. (d) Immunoblot showing the molecular masses of CSF1 proteins (pointed by yellow arrowheads) in conditioned media and cell lysates. (e-g) Immunostaining for GFP and CSF1 in a spinal cord section from 1 week post injection of LV/hSyn_CSF1-2A-GFP showed that GFP and CSF1 were expressed in the same neurons and their neurites. Scale bar in $(a)=50 \mu \mathrm{m}$, also applies to (b-c); scale bar in (e) $=50 \mu \mathrm{m}$, also applies to (f-g). 
FIGURE 11 Engineered over-expression of CSF1 in spinal neurons leads to microglial proliferation and enwrapping of $\mathrm{CSF}^{+}{ }^{+}$neurons. Spinal cord cross sections from mice at 1,2 , and 3 weeks post injection of LV/hSyn_GFP or LV/hSyn_CSF1-2A-GFP were immunostained for GFP and Iba1. (a-d) Spinal cord transduced by LV/hSyn_GFP at 1 week post injection; (e-h) 2 weeks post injection; (i-l) 3 weeks post injection. (m-p) Spinal cord transduced by LV/hSyn_CSF1-2A-GFP at 1 week post injection; (q-t) 2 weeks post injection; (u-x) 3 weeks post injection. Clusters of $\mathrm{Iba}^{+}$microglia are marked by * in (r). Scale bar in (a) $=50 \mu \mathrm{m}$, applies to the other panels. LV/GFP: LV/hSyn_GFP; LV/CSF1-GFP: LV/hSyn_CSF1-2A-GFP.

Figure 12 Engineered over-expression of CSF1 in spinal neurons leads to the loss of the large transduced neurons. Spinal cord cross sections from mice sacrificed at 1, 2, and 3 weeks post injection of LV/hSyn_GFP or LV/hSyn_CSF1-2A-GFP were immunostained for GFP and Iba1. Large $\mathrm{GFP}^{+}$neurons with areas of somota $>350 \mu \mathrm{m}^{2}$ and with DAPI-stained nuclei in the ventral horns were counted. Values are means \pm SD. $* * * p<0.001$, one-way ANOVA with post-hoc Tukey test, $\mathrm{n}=6$. LV/GFP: LV/hSyn_GFP; LV/CSF1-GFP: LV/hSyn_CSF12A-GFP.

Figure 13 Microglia are closely associated with neurites of CSF1-GFP transduced neurons. Spinal cord sections from mice sacrificed at 1 week post injection of LV/hSyn_GFP or LV/hSyn_CSF1-2A-GFP were immunostained for GFP and Iba1. (a-d) Iba1 ${ }^{+}$microglia were not associated with LV/hSyn_GFP transduced neurons and their neurites. (e-h) and (i-l) Iba1 ${ }^{+}$ microglia enwrapped the LV/hSyn_CSF1-2A-GFP-transduced neurons and were closely 
associated with the CSF1-GFP-expressing neurites. Scale bars in (a), (e) and (i) $=50 \mu \mathrm{m}$. LV/GF: LV/hSyn_GFP; LV/CSF1-GFP: LV/hSyn_CSF1-2A-GFP.

Figure 14 Engineered over-expression of CSF1 in spinal neurons leads to demyelination of $\mathrm{CSF}^{+}$axons. Cross sections of spinal cords from mice sacrificed at 3 weeks post injection of LV/hSyn_GFP (a-d) or LV/hSyn_CSF1-2A-GFP (e-f) were immunostained for GFP and MAG. * in (e) marks the area transduced by LV/hSyn_CSF1-2A-GFP, in (f) marks the same area with reduced MAG immunoreactivity, in (g) marks the same area with high density of cells demonstrated by nuclear staining. (i) Quantification of MAG immunoreactivities in areas transduced by LV/hSyn_GFP or LV/hSyn_CSF1-2A-GFP compared with the adjacent untransduced areas. ${ }^{* * *} p<0.001$, one-way ANOVA with post-hoc Tukey test, $\mathrm{n}=6$. (j-o) Two groups of confocal images showing that the MAG immunoreactivity was closely associated with LV/hSyn_GFP transduced axons (white arrows in (1) and (o)). (p-u) Two groups of confocal images showing that the MAG immunoreactivity was not associated with LV/hSyn_CSF1-2A-GFP transduced axons. Scale bar in (a) $=100 \mu \mathrm{m}$, also applies to (b-h). Scale bar in $(j)=5 \mu \mathrm{m}$, also applies to $(\mathrm{k}-\mathrm{u})$. MAG-ir: MAG immunoreactivity. Untrans: untransduced; Trans: transduced. LV/GFP: LV/hSyn_GFP; LV/CSF1-GFP: LV/hSyn_CSF12A-GFP. 
TABLE 1 Sources and concentrations of primary antibodies used for immunohistochemistry

\begin{tabular}{|l|l|l|l|l|l|}
\hline Molecules & $\begin{array}{l}\text { Host } \\
\text { species }\end{array}$ & Sources & $\begin{array}{l}\text { Catalogue } \\
\text { numbers }\end{array}$ & RRID & Dilution \\
\hline Mouse CSF1 & Goat & PeproTech & 500-P62G & - & $1: 200$ \\
\hline Mouse CSF1 & Goat & R\&D Systems & AF416 & AB_355351 & $1: 200$ \\
\hline GFAP & Rabbit & Dako & Z0334 & AB_10013382 & $1: 500$ \\
\hline NeuN & Rabbit & Abcam & ab177487 & AB_2532109 & $1: 200$ \\
\hline Iba1 & Rabbit & Wako & $019-19741$ & AB_839504 & $1: 400$ \\
\hline Iba1 & Goat & Novus & NB100-1028 & AB_521594 & $1: 400$ \\
\hline TMEM119 & Rabbit & Abcam & Ab185333 & AB_2687894 & $1: 200$ \\
\hline MAG & Rabbit & Thermo Fisher & $34-6200$ & AB_87330 & $1: 100$ \\
\hline GFP & Rabbit & Abcam & Ab290 & AB_303395 & $1: 1000$ \\
\hline GFP & Sheep & BioRad & $4745-1051$ & AB_619712 & $1: 500$ \\
\hline CD3 & Rabbit & Novus & NB600-1441 & AB_789102 & $1: 200$ \\
\hline
\end{tabular}


(a)

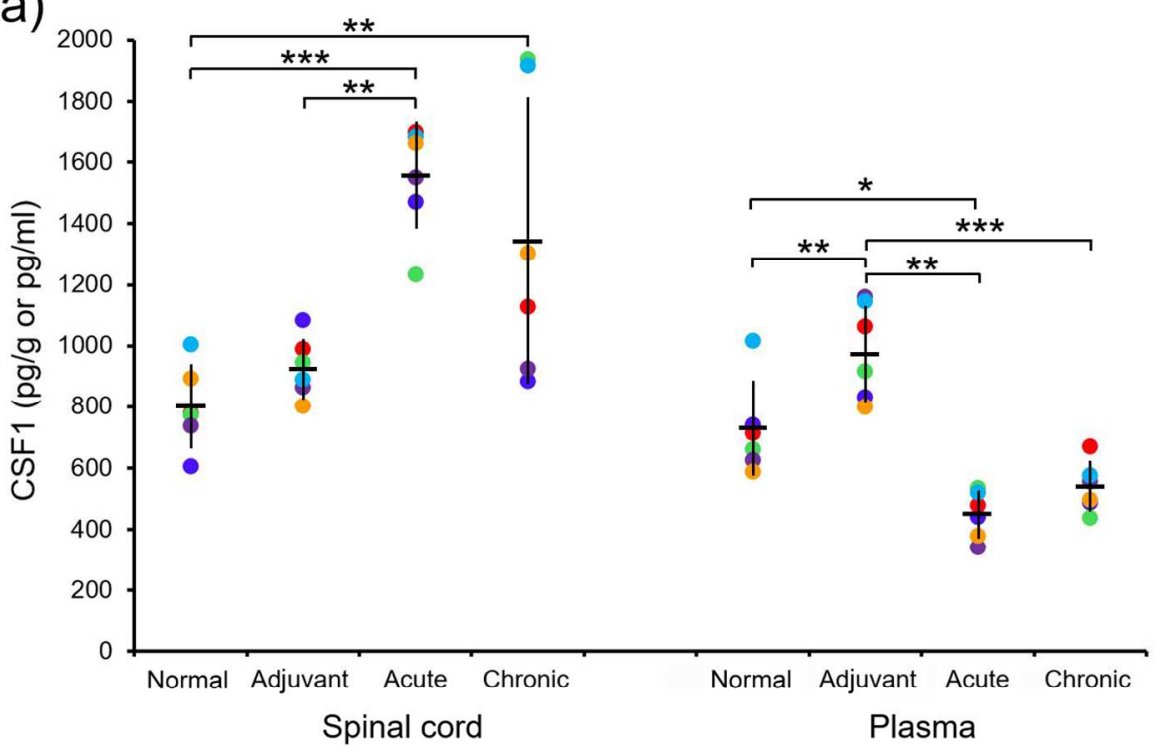

(b)
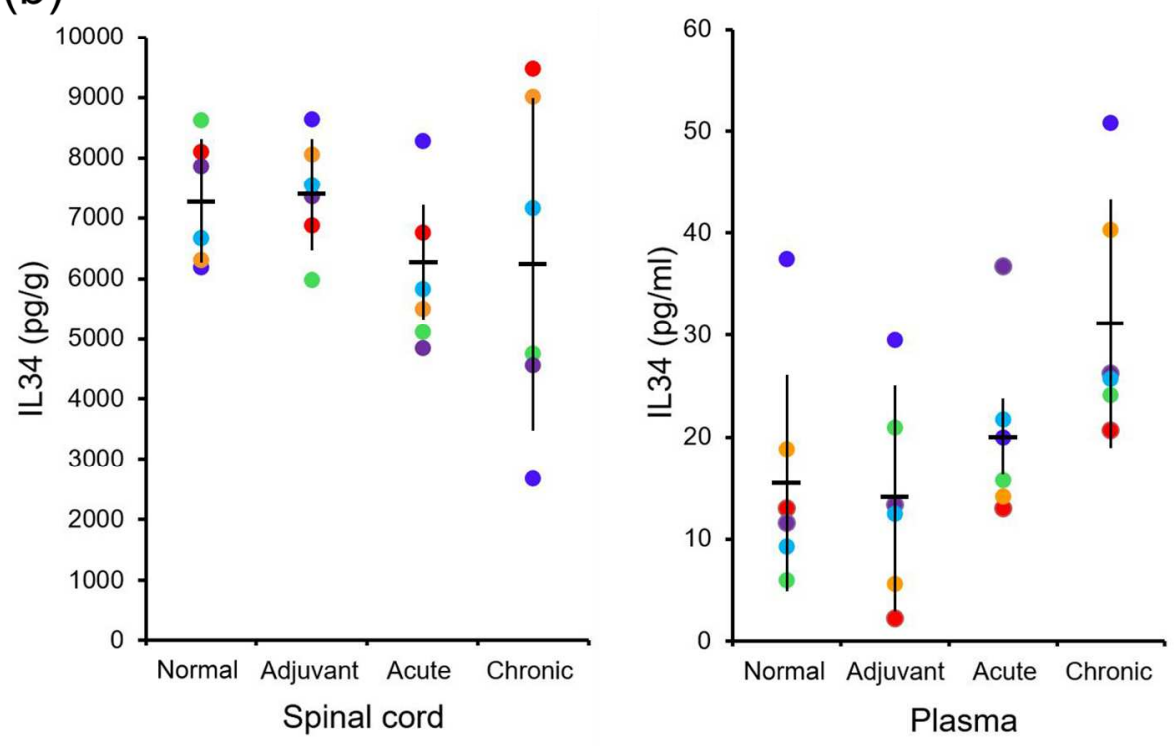

FIGURE 1 Protein levels of CSF1 and IL34 in mouse spinal cords and plasma $114 \times 151 \mathrm{~mm}(300 \times 300 \mathrm{DPI})$ 


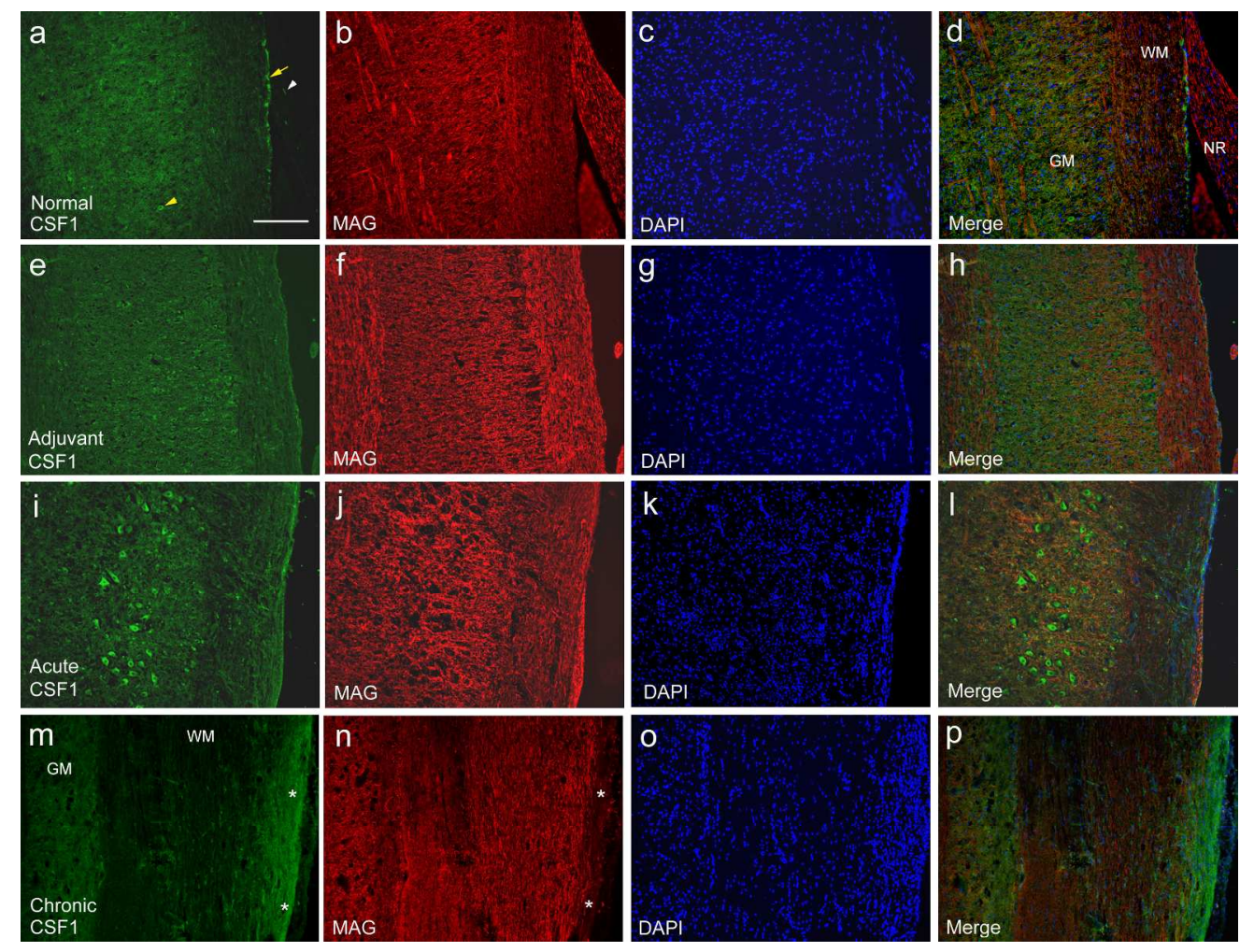

FIGURE 2 CSF1 expression increases in the spinal cords of acute and chronic EAE $244 \times 186 \mathrm{~mm}(300 \times 300$ DPI) 

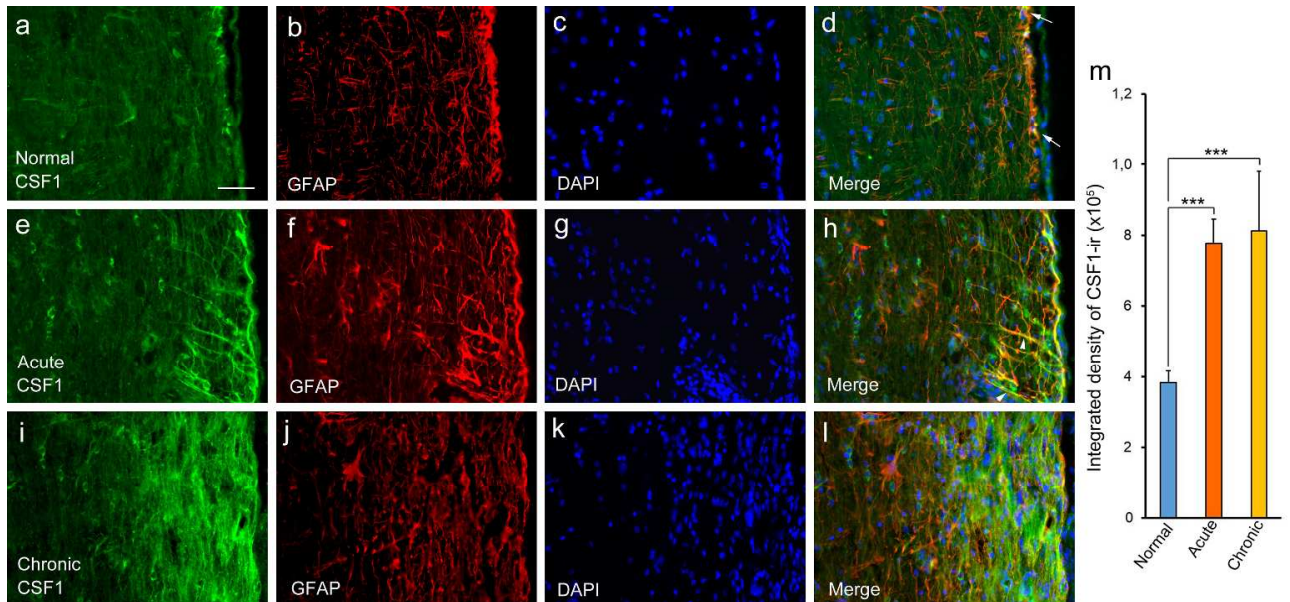

DAPI

FIGURE 3 CSF1 expression in astrocytes in the white matter increases significantly in acute and chronic EAE mice

$299 \times 138 \mathrm{~mm}(300 \times 300 \mathrm{DPI})$ 

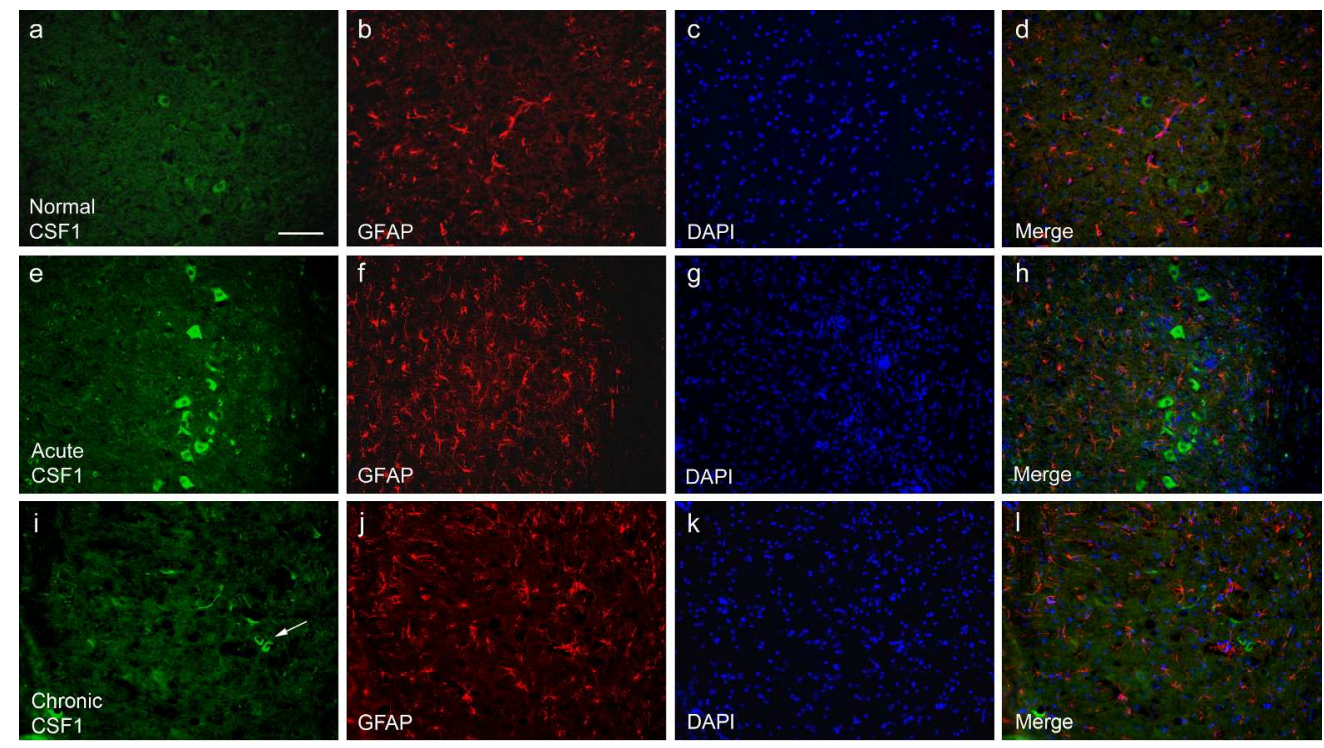

FIGURE 4 CSF1 expression in astrocytes in the gray matter does not increase significantly in acute and chronic EAE mice

$244 \times 137 \mathrm{~mm}(300 \times 300 \mathrm{DPI})$ 

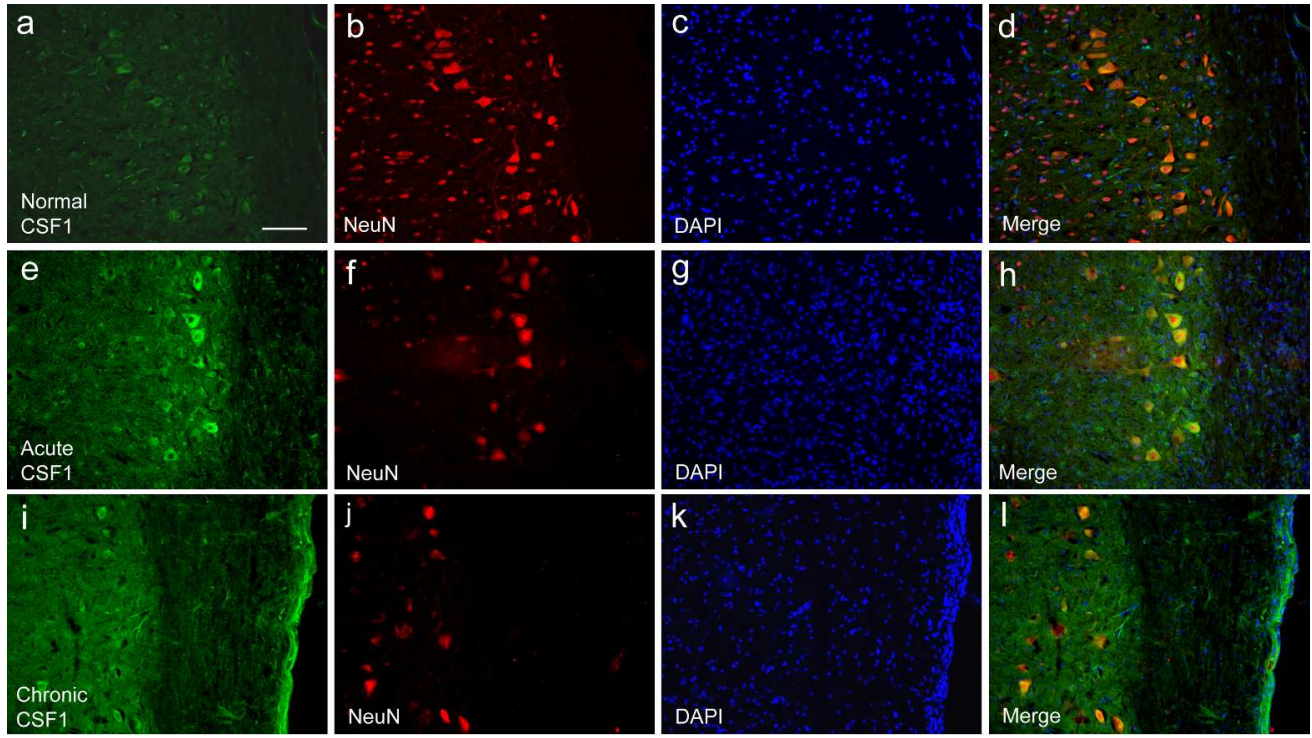

FIGURE 5 CSF1 expression increases significantly in spinal neurons of acute and chronic EAE mice. $243 \times 136 \mathrm{~mm}(300 \times 300 \mathrm{DPI})$ 


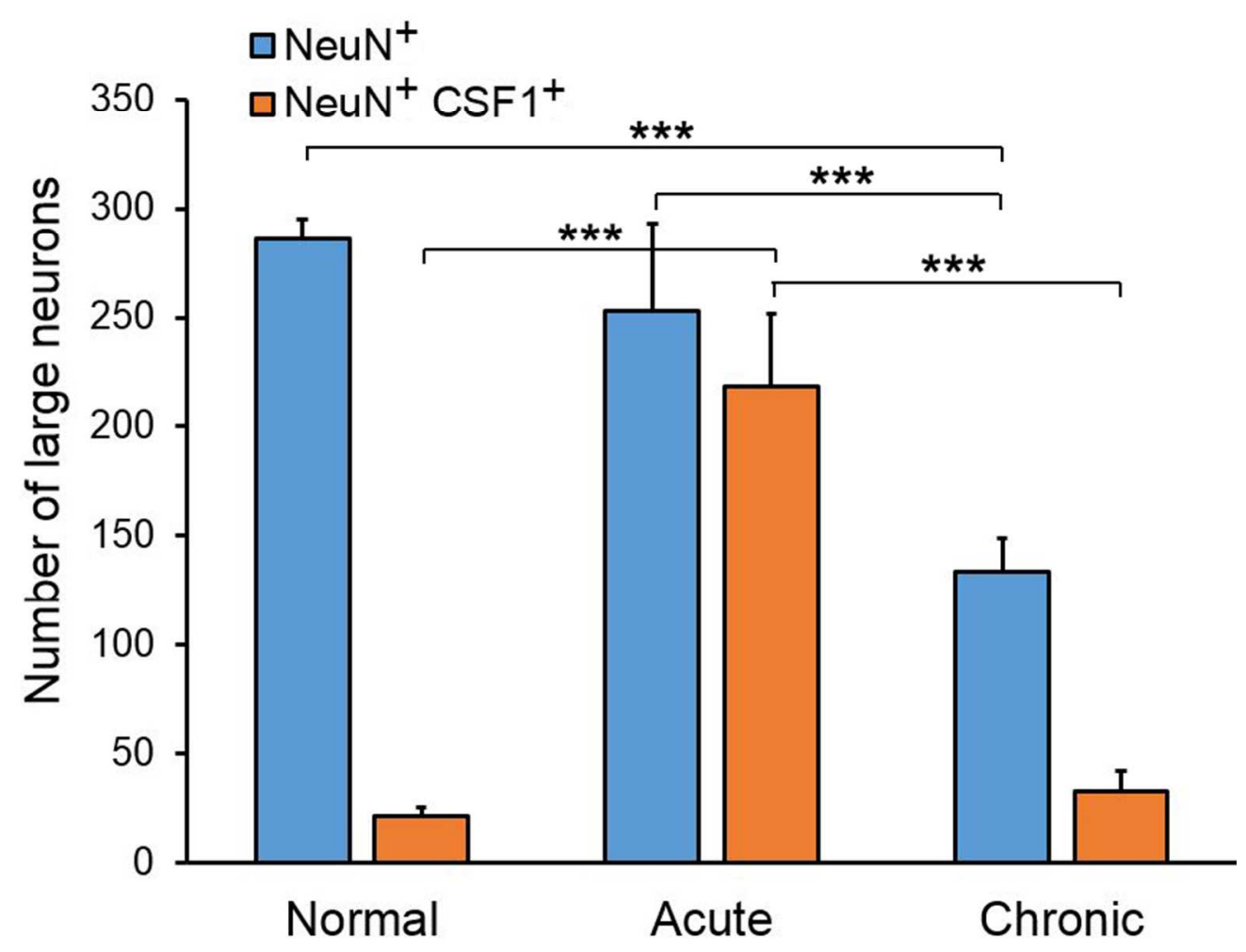

FIGURE 6 Quantification of the number of large spinal motoneurons and the number of large motoneurons with high-level CSF1 expression.

$180 \times 143 \mathrm{~mm}(120 \times 120 \mathrm{DPI})$ 

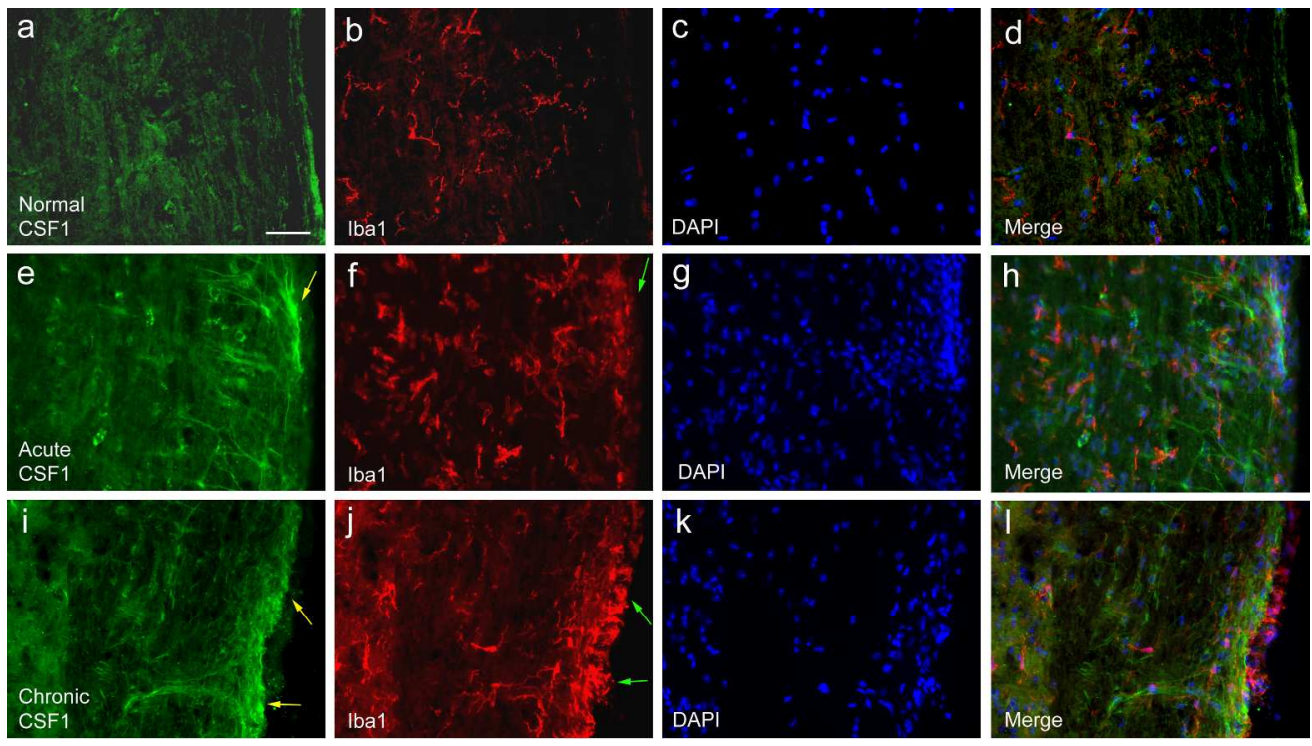

FIGURE 7 High-level CSF1 expression correlates with high densities of microglia in acute and chronic EAE mice.

$245 \times 137 \mathrm{~mm}(300 \times 300 \mathrm{DPI})$ 


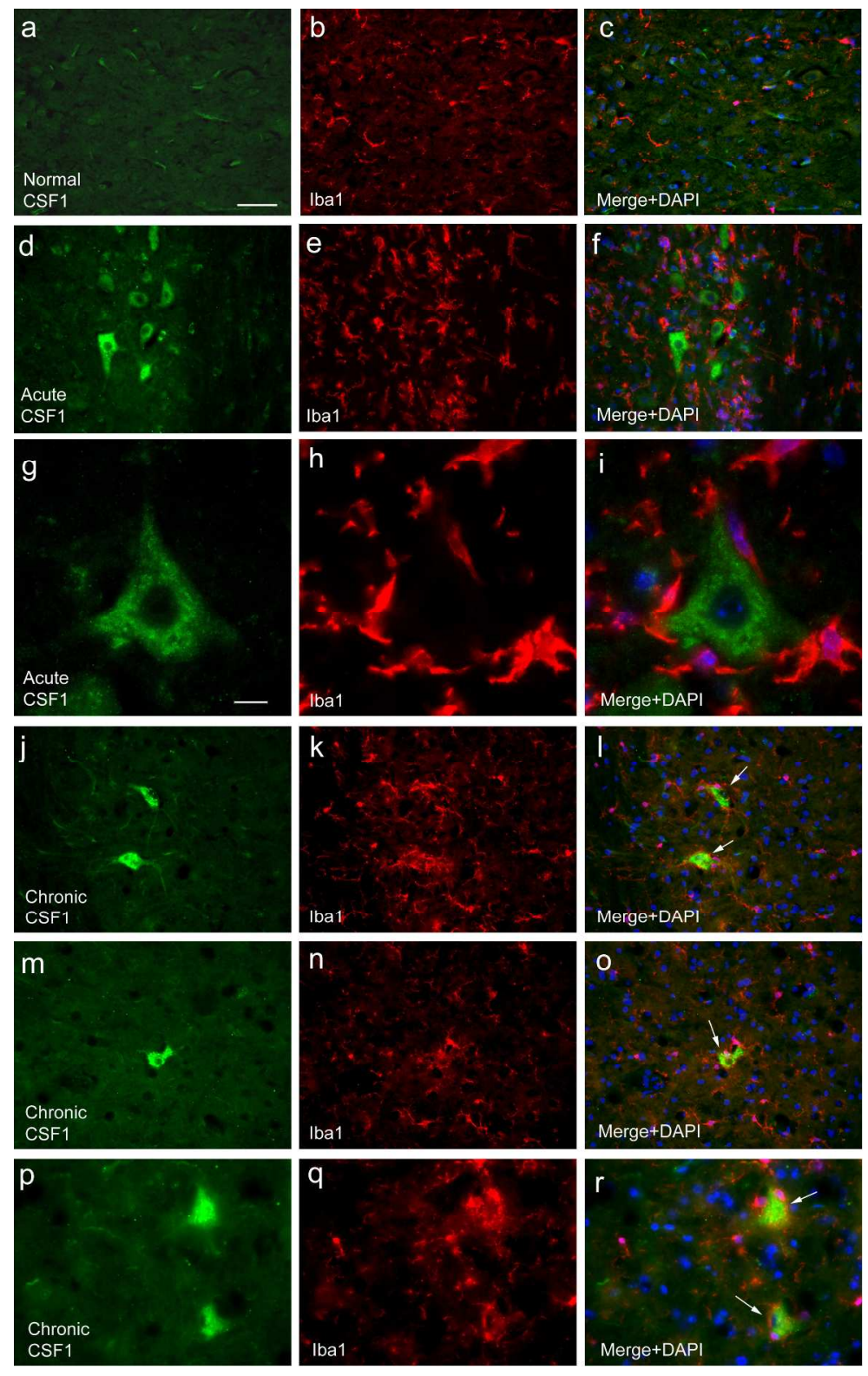

FIGURE 8 Microglia surround the neurons with high-level CSF1 expression in the spinal cords of acute and chronic EAE mice.

$183 \times 293 \mathrm{~mm}(300 \times 300$ DPI $)$ 

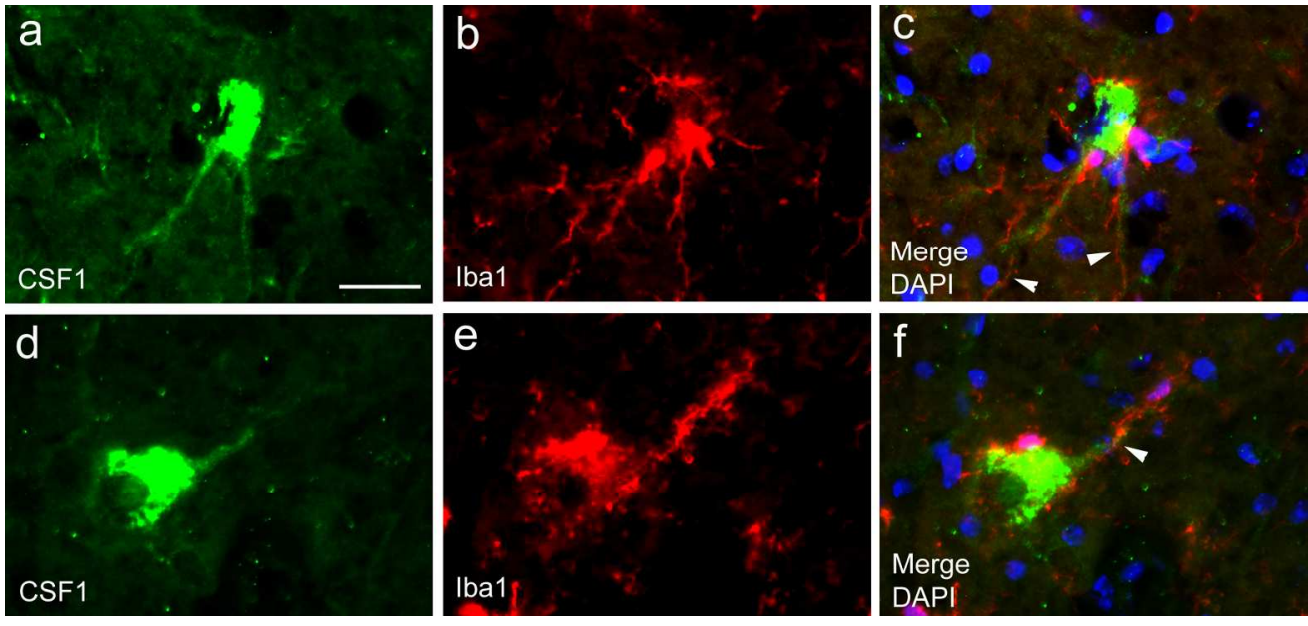

FIGURE 9 Microglia are closely associated with CSF1+ neurites in the spinal cord of chronic EAE mice. $182 \times 85 \mathrm{~mm}(300 \times 300 \mathrm{DPI})$ 

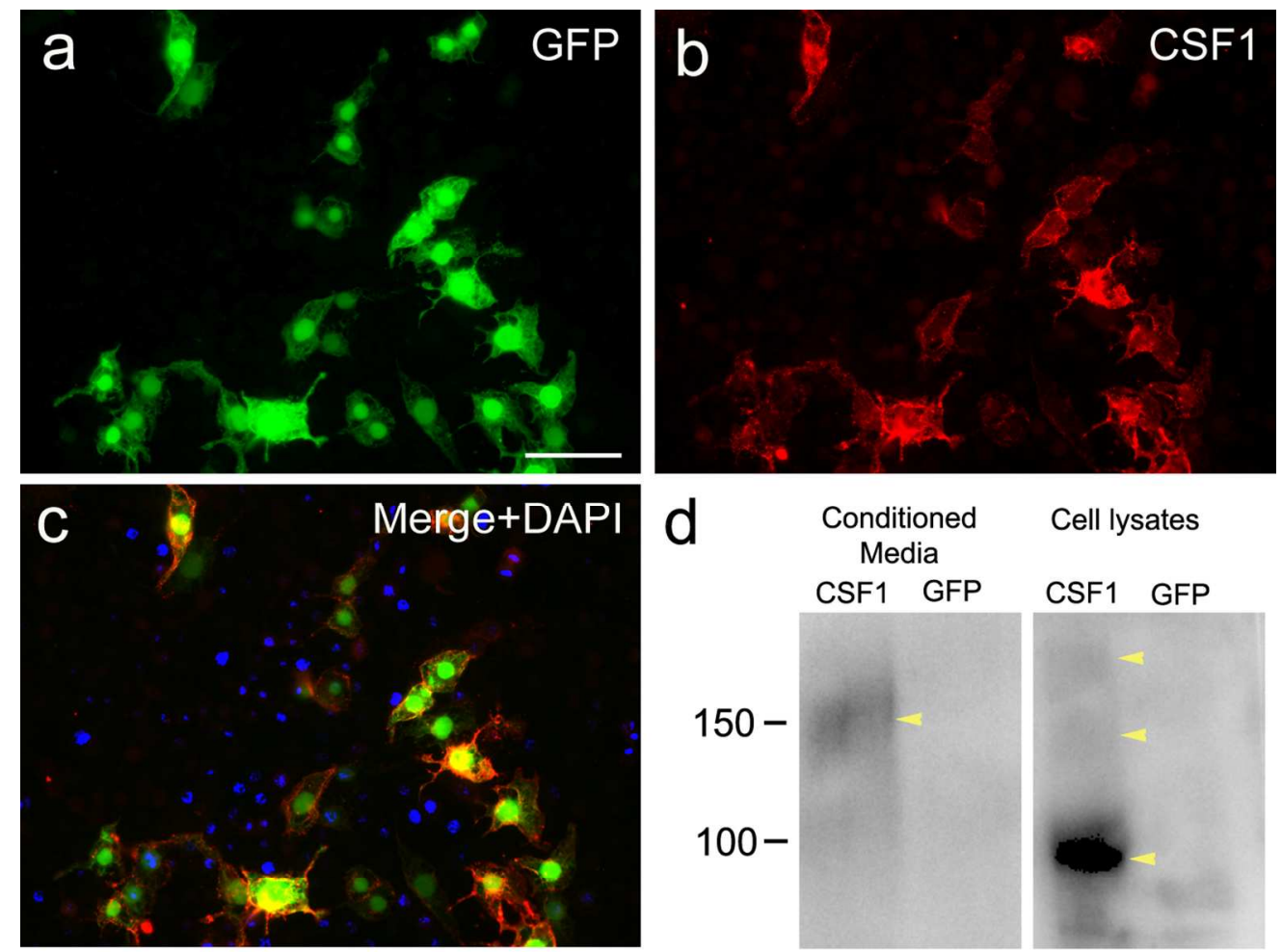

\section{Conditioned}

Cell lysates Media
CSF1 GFP CSF1 GFP
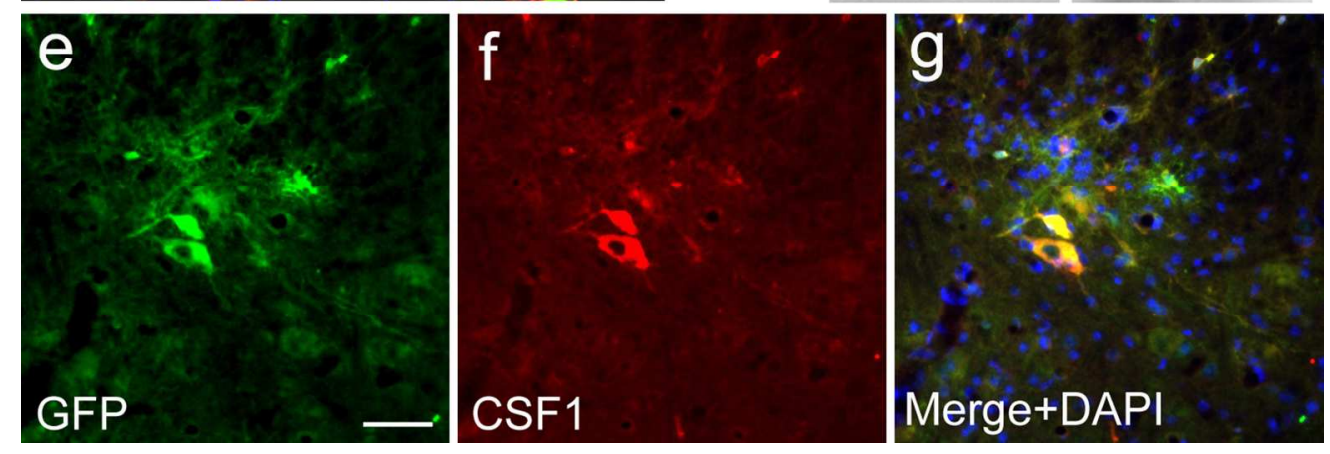

FIGURE 10 Engineered over-expression of CSF1 in vitro and in vivo.

$121 \times 131 \mathrm{~mm}(300 \times 300 \mathrm{DPI})$ 

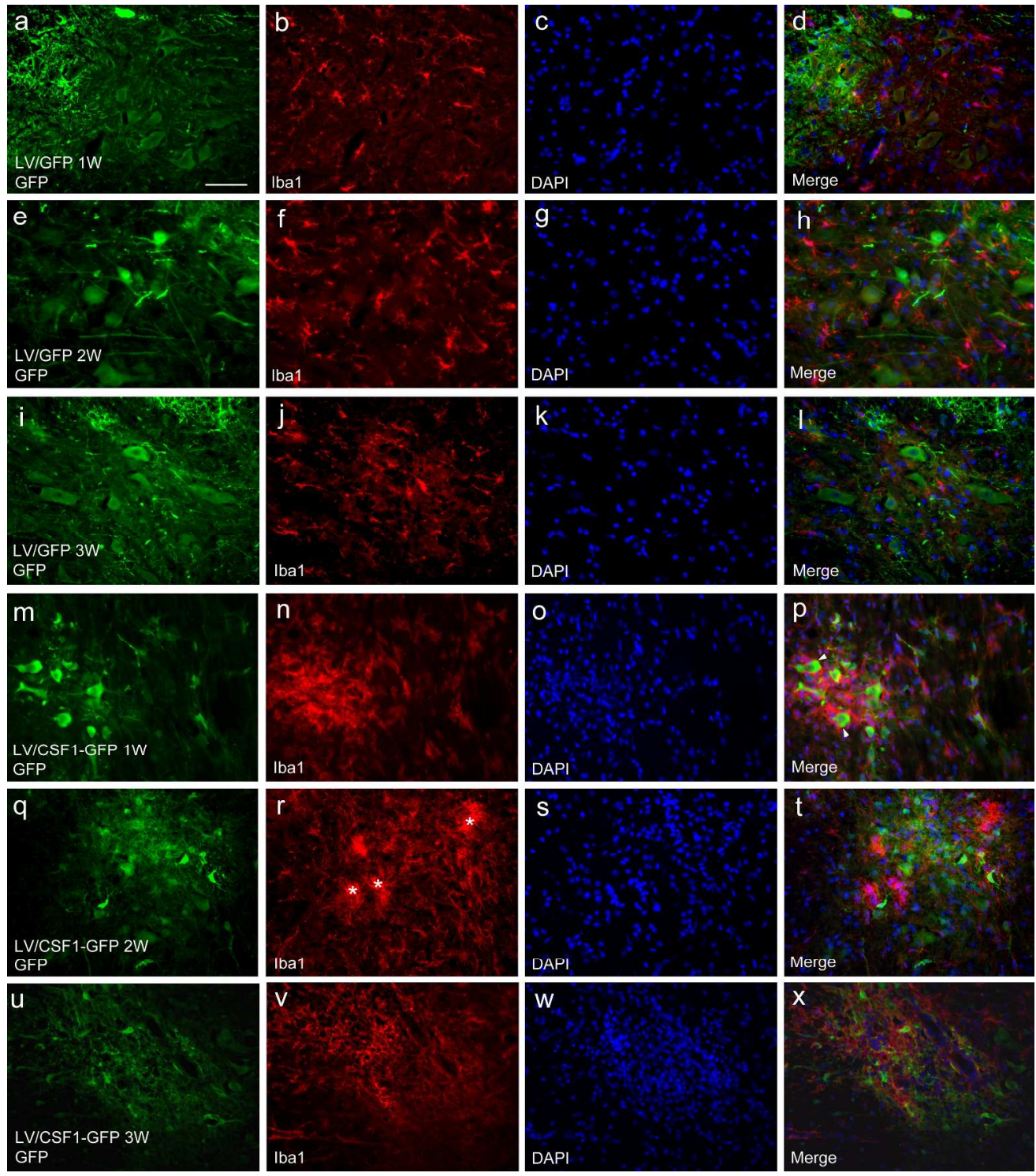

FIGURE 11 Engineered over-expression of CSF1 in spinal neurons leads to microglial proliferation and enwrapping of CSF1+ neurons.

$244 \times 277 \mathrm{~mm}(300 \times 300$ DPI) 


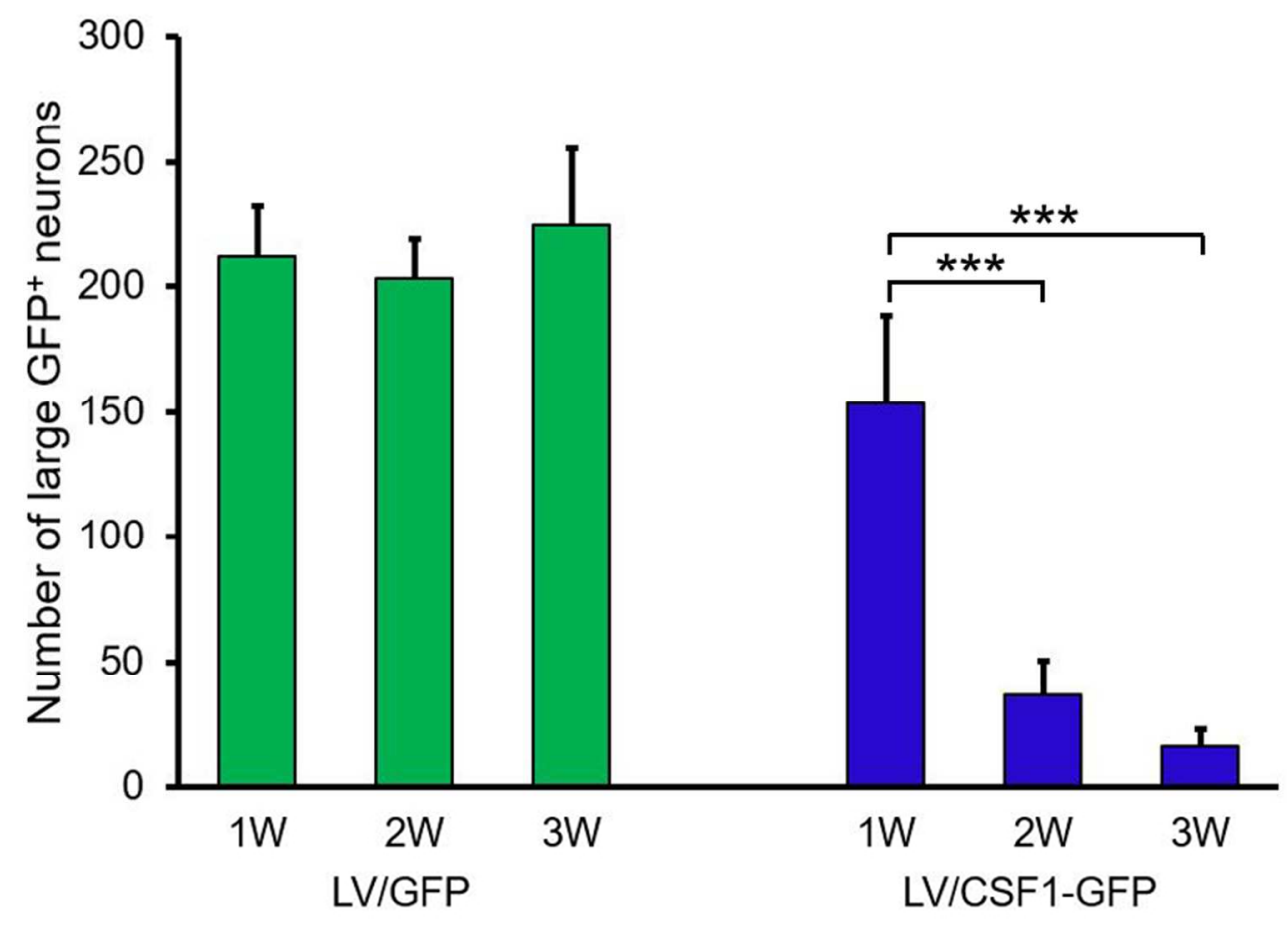

Figure 12 Engineered over-expression of CSF1 in spinal neurons leads to the loss of the large transduced neurons.

$75 \times 55 \mathrm{~mm}(300 \times 300$ DPI $)$ 

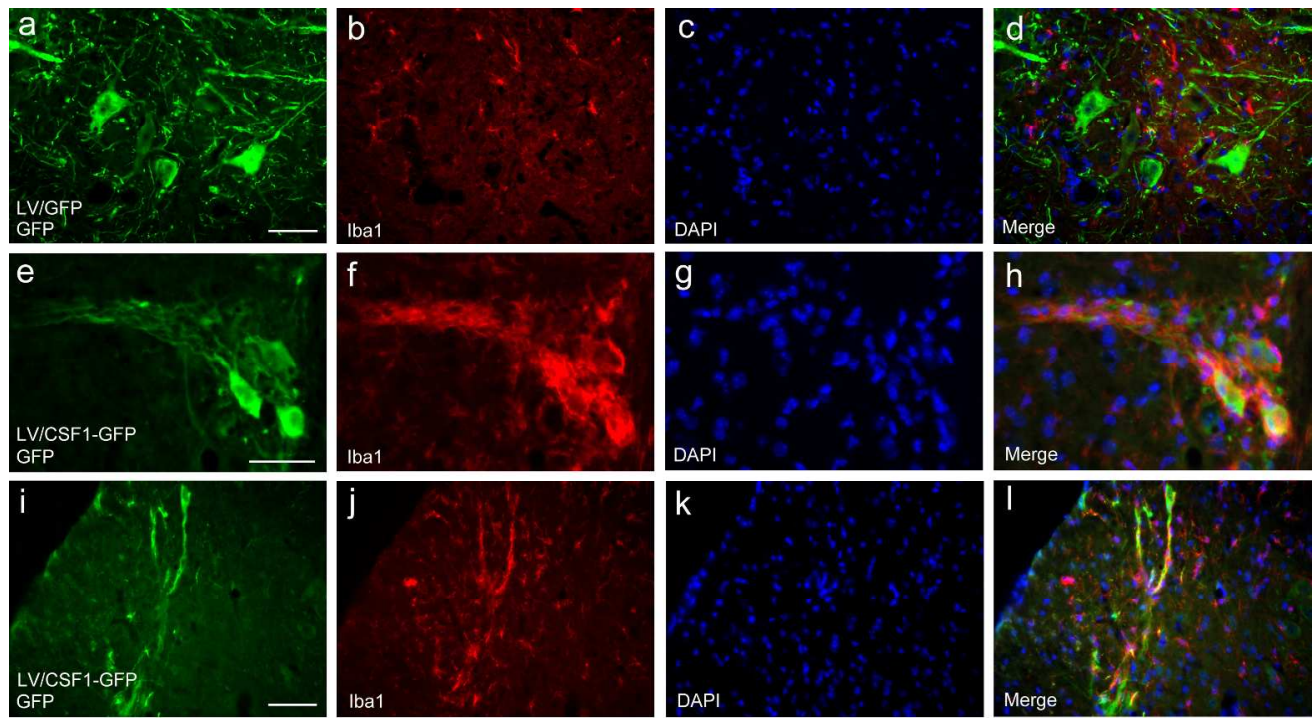

Figure 13 Microglia are closely associated with neurites of CSF1-GFP transduced neurons. $246 \times 134 \mathrm{~mm}(300 \times 300 \mathrm{DPI})$ 

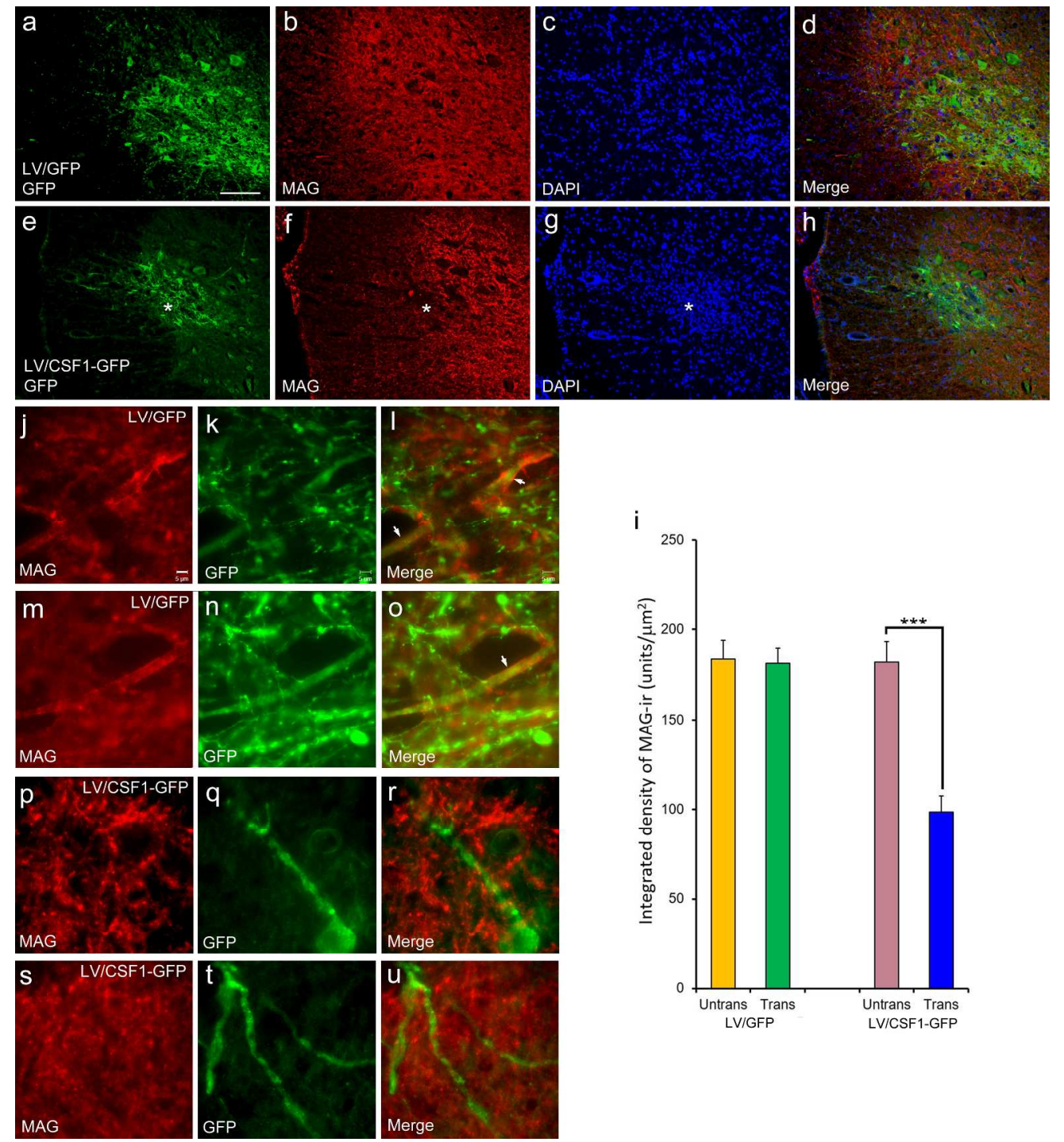

Figure 14 Engineered over-expression of CSF1 in spinal neurons leads to demyelination of CSF1+ axons. $243 \times 266 \mathrm{~mm}(300 \times 300 \mathrm{DPI})$ 


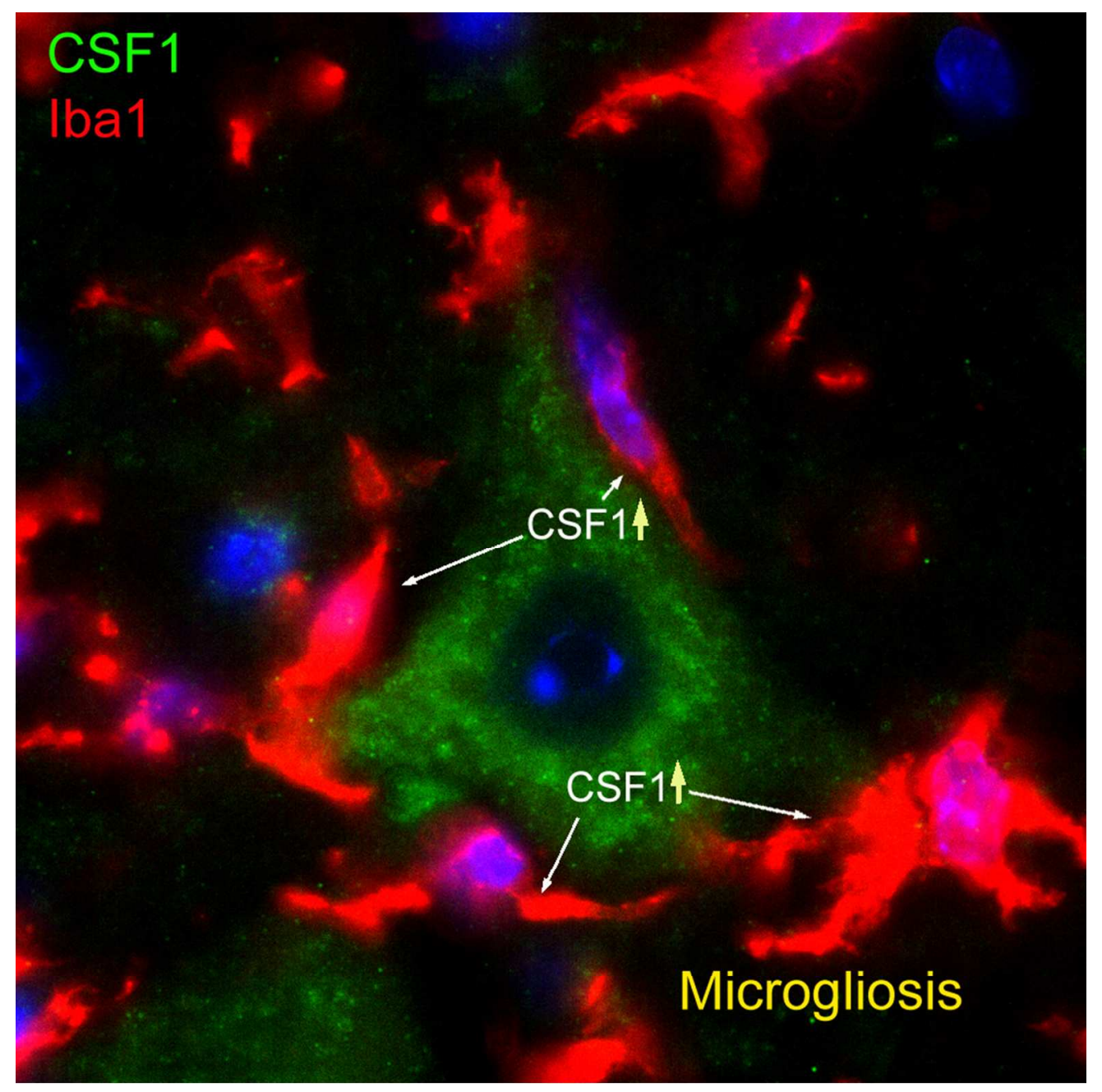

Table of Contents image

$82 \times 82 \mathrm{~mm}(300 \times 300 \mathrm{DPI})$ 

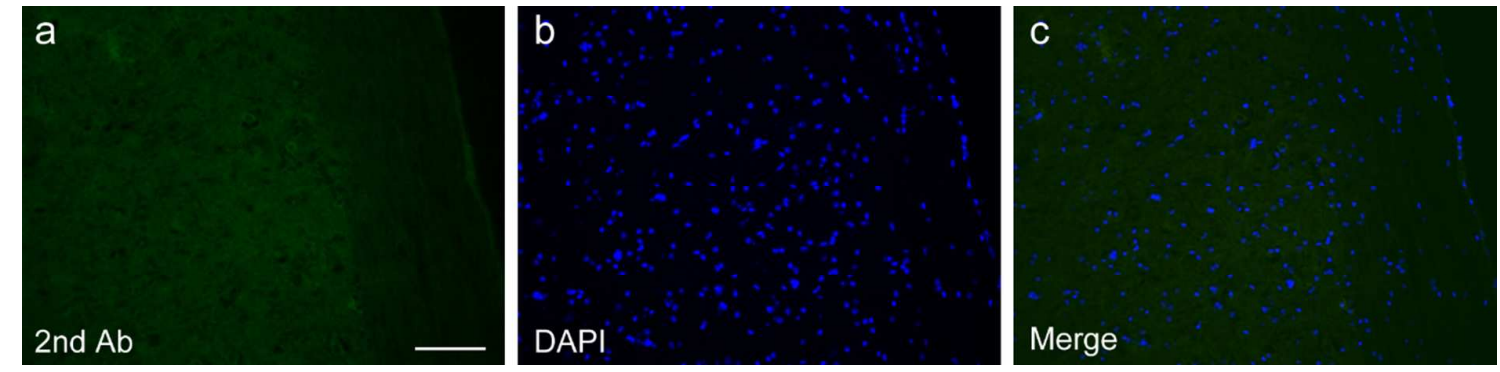

Figure S1 Negative control for CSF1 immunotostaining. (a) Immunostaining of a horizontal spinal cord section from a normal ABH mouse in the absence of anti-CSF1 antibody. (b) DAPI staining of nuclei. (c) Merged image of (a) and (b). Scale bar $=100 \mu \mathrm{m}$.
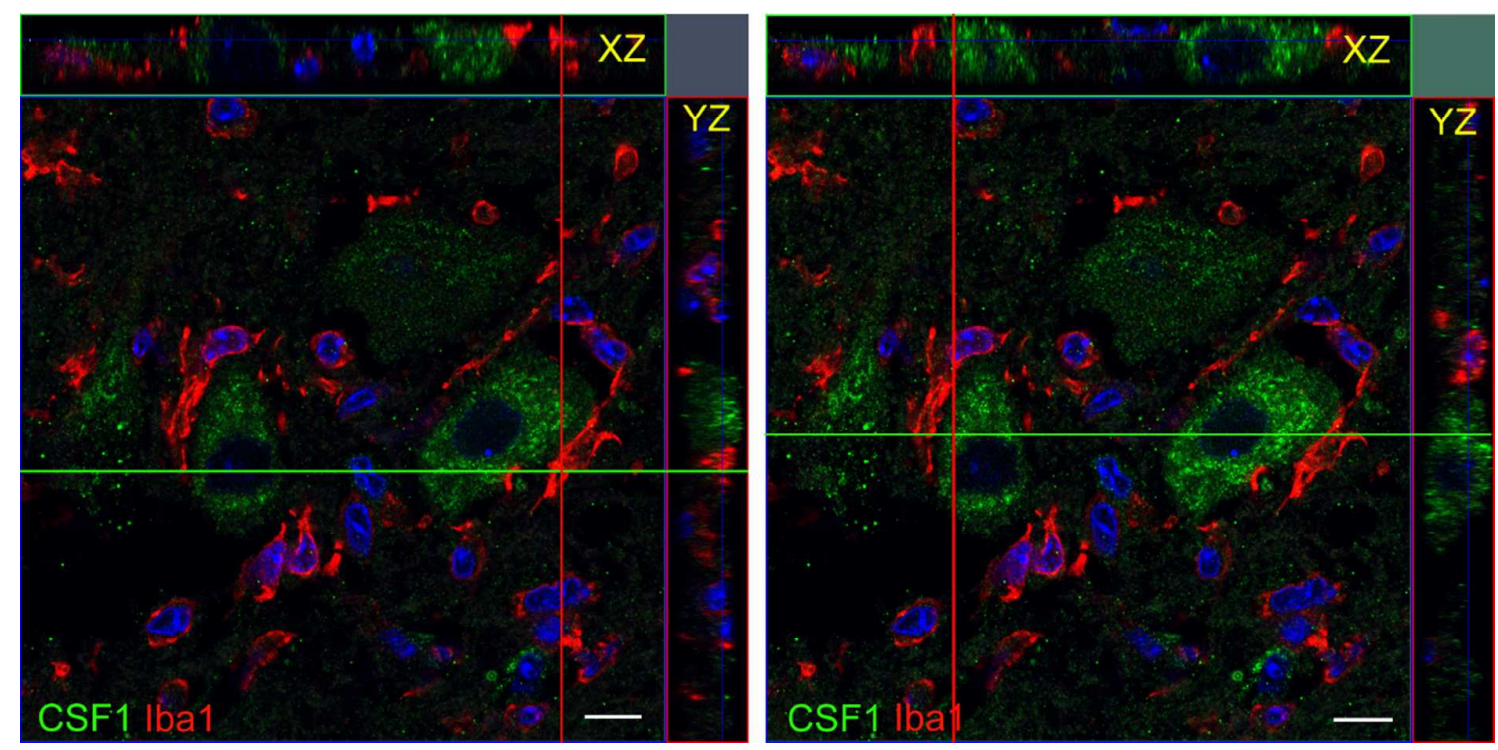

Figure S2 CSF1 immunoreactivity is not observed in microglia. Confocal microscopy images of a CSF1- and Iba1-double immunostained section of acute EAE mouse spinal cord were captured by a Zeiss LSM 710 confocal microscope using a $63 \times$ oil objective. Images were taken at a z-stack of 34 sections with a section interval of $0.348 \mu \mathrm{m}$. Scale bar $=10 \mu \mathrm{m}$ 

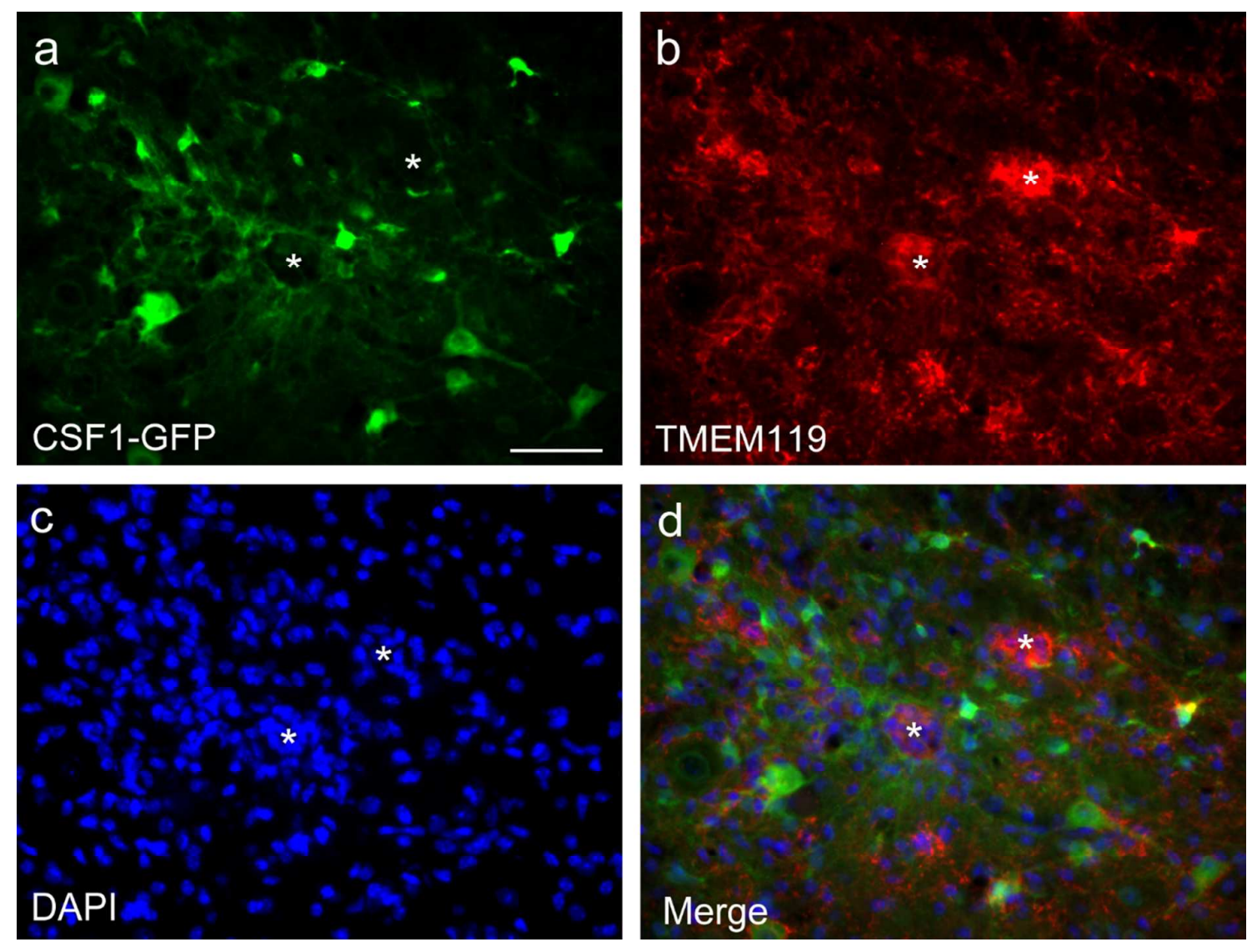

Figure S3 Clusters of microglia in mouse spinal cord. A cross-section of a mouse spinal cord two weeks after LV/hSyn_CSF1-2A-GFP injection was stained with anti-TMEM119 and anti-GFP antibodies. Clusters of TMEM119 ${ }^{+}$microglia are marked by *. Scale bar $=100 \mu \mathrm{m}$. 

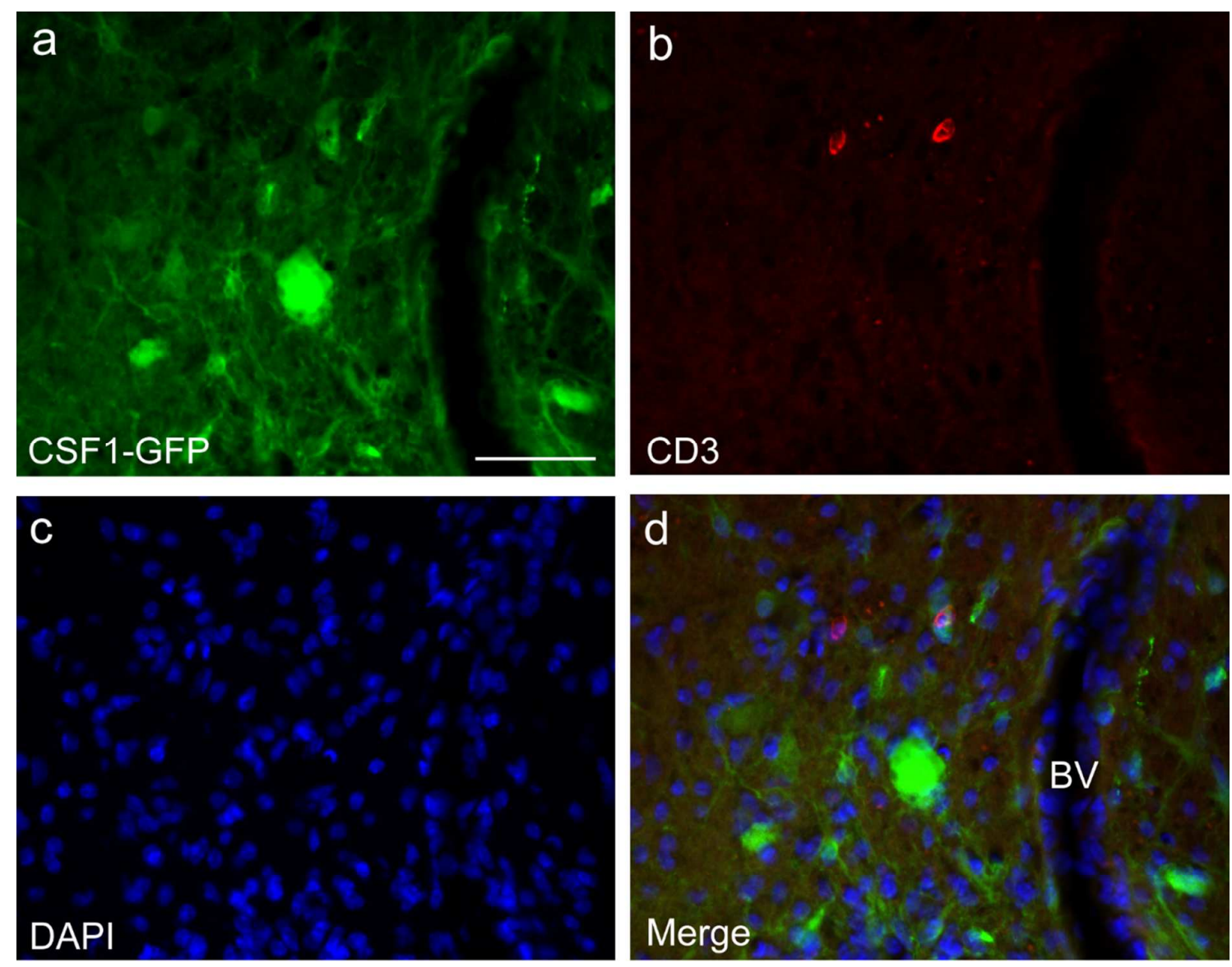

Figure S4 Over-expression of CSF1 in mouse spinal cord does not lead to aggregation of T cells around the transduced neurons. To assess whether T-cells contribute to loss of CSF1GFP-transduced large motoneurons in spinal cord, double immunostaining for CD3 and GFP was performed on spinal cord sections from mice one week after LV/hSyn_CSF1-2A-GFP injection. Antigen retrieval was performed by heating the sections at $80^{\circ} \mathrm{C}$ for $30 \mathrm{~min}$ in Antigen Retrieval Buffer (pH6.0), then cooled for 20 min at room temperature before applying blocking buffer. A sheep anti-GFP and a rabbit anti-CD3 monoclonal antibody were applied and incubated overnight at room temperature. (a) $\mathrm{GFP}^{+}$neurons and axons representing CSF1 expression in viral vector transduced neurons; (b) $\mathrm{CD}^{+}{ }^{+}$-cells; (c) nuclei stained with DAPI; (d) merged image. BV, blood vessel. Scale bar $=50 \mu \mathrm{m}$. 